\title{
Observed acidification trends in North Atlantic water masses
}

\author{
M. Vázquez-Rodríguez ${ }^{1}$, F. F. Pérez ${ }^{1}$, A. Velo ${ }^{1}$, A. F. Ríos ${ }^{1}$, and H. Mercier ${ }^{2}$ \\ ${ }^{1}$ Department of Oceanography, Instituto de Investigaciones Marinas (IIM), CSIC, Eduardo Cabello 6, 36208 Vigo, Spain \\ ${ }^{2}$ Laboratoire de Physique des Océans (LPO), CNRS Ifremer IRD UBO, IFREMER Centre de Brest, \\ B.P. 7029280 Plouzané, France \\ Correspondence to: M. Vázquez-Rodríguez (mvazquez@iim.csic.es)
}

Received: 29 February 2012 - Published in Biogeosciences Discuss.: 14 March 2012

Revised: 8 November 2012 - Accepted: 26 November 2012 - Published: 18 December 2012

\begin{abstract}
The lack of observational $\mathrm{pH}$ data has made it difficult to assess recent rates of ocean acidification, particularly in the high latitudes. Here we present a time series that spans over 27 yr (1981-2008) of high-quality carbon system measurements in the North Atlantic, which comprises fourteen cruises and covers the important water mass formation areas of the Irminger and Iceland Basins. We provide direct quantification of acidification rates in upper and intermediate North Atlantic waters. The highest rates were associated with surface waters and with Labrador Sea Water (LSW). The Subarctic Intermediate and Subpolar Mode Waters (SAIW and SPMW) showed acidification rates of $-0.0019 \pm 0.0001$ and $-0.0012 \pm 0.0002 \mathrm{yr}^{-1}$, respectively. The deep convection activity in the North Atlantic Subpolar Gyre injects surface waters loaded with anthropogenic $\mathrm{CO}_{2}$ into lower layers, provoking the remarkable acidification rate observed for LSW in the Iceland Basin $\left(-0.0016 \pm 0.0002 \mathrm{yr}^{-1}\right)$. An extrapolation of the observed linear acidification trends suggests that the $\mathrm{pH}$ of LSW could drop 0.45 units with respect to pre-industrial levels by the time atmospheric $\mathrm{CO}_{2}$ concentrations reach $\sim 775 \mathrm{ppm}$. Under circulation conditions and evolution of $\mathrm{CO}_{2}$ emission rates similar to those of the last three decades, by the time atmospheric $\mathrm{CO}_{2}$ reaches $550 \mathrm{ppm}$, an aragonite undersaturation state could be reached in the cLSW of the Iceland Basin, earlier than surface SPMW.
\end{abstract}

\section{Introduction}

The ocean acidification due to the increasing atmospheric $\mathrm{CO}_{2}$ is a well-known fact (Bates et al., 2012; Doney et al., 2009; Raven, 2005) but the direct $\mathrm{pH}$ observations are sparse
(Byrne et al., 2010; Tittensor et al., 2010; Wootton et al., $2008)$. Roughly $20-35 \%$ of the anthropogenic $\mathrm{CO}_{2}\left(\mathrm{C}_{\text {ant }}\right)$ emissions are absorbed by the oceans (Khatiwala et al., 2009) and mitigate global warming. Since the beginning of the Industrial Revolution, the sea surface has seen a $30 \%$ increase in hydrogen ion concentrations $\left[\mathrm{H}^{+}\right]$(Caldeira and Wickett, 2005; Raven, 2005). The current acidification episode is occurring $\sim 100$ times faster than any other acidity change in the last 50 million yr of Earth's history (Pelejero et al., 2010).

Ocean acidification is thought to be the onset for a number of cascading effects throughout marine ecosystems that may leave no time for many organisms to adapt, especially in the case of calcareous organisms (Feely et al., 2008; Doney et al., 2009). It causes a combination of contrasted impacts on the marine environment, from reproductive larval survivorship and growth-related issues in several taxa (Doney et al., 2009) to the reduction of seawater's sound absorption coefficient (Ilyina et al., 2009). Cold-water scleractinian corals dwelling in intermediate and deep North Atlantic (NA) waters are particularly vulnerable to acidification (Guinotte et al., 2006; Raven, 2005).

The North Atlantic Subpolar Gyre (NASPG) is an important formation area of mode waters. These waters, formed in deep winter mixed layers, are identified by nearly uniform properties in the vertical, near the top of the permanent pycnocline (Thierry et al., 2008). The process of transformation of the warm, saline subtropical waters into intermediate and deep waters in the NASPG (McCartney and Talley, 1982; Read, 2001) results in several varieties of Subpolar Mode Water (SPMW) distributed around the gyre. The Labrador Sea Water (LSW), the densest variety of SPMW, is one of the thickest water masses in the NA and one of the main components of the lower limb of the Meridional Overturning 
Circulation (Thierry et al., 2008). The LSW has high contents of chlorofluorocarbons (CFCs) and anthropogenic carbon due to ventilation processes (Azetsu-Scott et al., 2003; Pérez et al., 2010). Thus, it is expected that those water masses will suffer changes in $\left[\mathrm{H}^{+}\right]$.

There are relatively few places where the carbon system has been surveyed thoroughly enough to generate a comprehensive database that can be used in the assessment of ocean acidification and its environmental impacts (Wootton et al., 2008). Several past and future $\mathrm{pH}$ projections have been proposed from Ocean General Circulation Models (GCMs) and model data (Orr et al., 2005), but in situ measurements documenting the evolution of ocean $\mathrm{pH}$ over time are limited (Wootton et al., 2008). The present work examines the temporal variability of $\mathrm{pH}$ in the main water masses of the North Atlantic using direct observations. Here we have gathered the available high-quality carbon system data that covered the NASPG between 1981 and 2008 (Fig. 1a) to study the decadal acidification rates of the main North Atlantic water masses (Fig. 1b).

\section{Dataset and methodology}

\subsection{Dataset}

A total of fourteen cruises with high-quality carbon system measurements were selected to follow the temporal evolution of $\mathrm{pH}$ in the North Atlantic (Fig. 1a, Table 1). The used cruise data can be accessed at CARINA site http://store. pangaea.de/Projects/CARBOOCEAN/carina/index.htm. We also used the climatological WOA05 data available at http: //www.nodc.noaa.gov/OC5/WOA05/pr_woa05.html.

Over time different analytical procedures were used to measure $\mathrm{pH}$, so different adjustments and corrections were applied to the raw data (Table 1) to create the $\mathrm{pH}$ dataset used in this study. The $\mathrm{pH}$ measurements in the database were determined either potentiometrically (using $\mathrm{pH}$ electrodes; Dickson, 1993) or, more commonly, with a spectrophotometric method that used m-cresol purple as a $\mathrm{pH}$ indicator in either scanning or diode array spectrophotometers (Clayton and Byrne, 1993). The spectrophotometric $\mathrm{pH}$ at sea has a reported analytical precision of approximately $0.0004 \mathrm{pH}$ units (Clayton and Byrne, 1993). Periodic checks of the $\mathrm{pH}$ measurement precision with Certified Reference Material (CRM) during the FOUREX and OVIDE cruises indicated a precision better than $0.002 \mathrm{pH}$ units. However, the uncertainties inherent to the use of equimolal tris buffers to obtain the constants used by Clayton and Byrne (1993) suggest a final uncertainty for spectrophotometric $\mathrm{pH}$ measurements of $\sim 0.004$, as reported in Table 1 . This value is consistent with our comparisons between the measured and the calculated $\mathrm{pH}$ using the acid dissociation constants in Dickson and Millero (1987) and reported in Clayton and Byrne (1993). All $\mathrm{pH}$ measurements that had not been originally reported
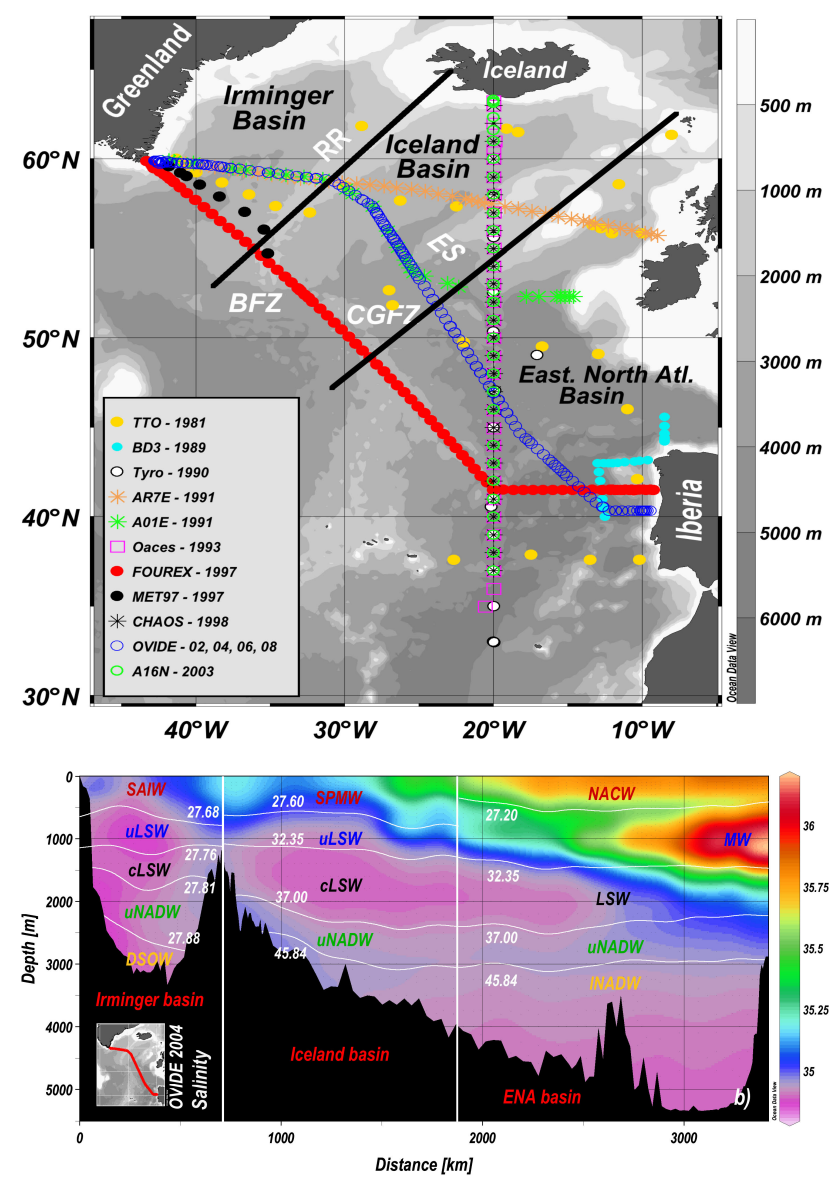

Fig. 1. (a) shows the study area and selected cruises. The thick black lines delimit the Irminger, Iceland and Eastern North Atlantic (ENA) Basins; (b) shows the main NASPG water masses considered for this study over the salinity distribution of the OVIDE 2004 section, which gives representative coverage of the NASPG. The vertical thick white lines delimit the Irminger, Iceland and ENA Basins. The isopycnals (horizontal white lines; $\sigma_{\theta}$, in $\mathrm{kg} \mathrm{m}^{-3}$ ) are the ones listed in Sect. 2.2 and Table 2. The water mass acronyms stand for: SAIW $=$ Subarctic Intermediate Water; LSW = Labrador Seawater; NADW = North Atlantic Deep Water; SPMW = Subpolar Mode Water; NACW = North Atlantic Central Water; MW = Mediterranean Water. The lowercase letters "c", "u" and "l" before the acronyms denote, respectively, "classical", "upper" and "lower" water mass types/branches.

in the seawater scale $\left(\mathrm{pH}_{\mathrm{SWS} 25}\right.$; Millero, 2007) were converted to it from either the total or the NBS pH scale using the corresponding acid dissociation constants (Dickson and Millero, 1987), following the CARINA database second quality control recommendations for $\mathrm{pH}$ data scale unification and cruise adjustments (Velo et al., 2010). The acid dissociation constants of $\mathrm{HF}$ or $\mathrm{HSO}_{4}^{-}$(Millero, 2007) were used to convert $\mathrm{pH}$ values originally reported in the total scale (those measured spectrophotometrically; Table 1) to the SWS scale. The pHs measured potentiometrically were all reported on the NBS scale and were converted to the SWS 
Table 1. List of selected North Atlantic cruises (Fig. 1a). Acronyms denote: P.I. = principal investigator; $\mathrm{S}=$ variable measured with spectrophotometric techniques; $\mathrm{P}=$ variable measured with potentiometric techniques; Calc $=\mathrm{pH}$ calculated from $\mathrm{C}_{\mathrm{T}}$ and $\mathrm{A}_{\mathrm{T}}$ using the thermodynamic equations of the carbon system (Dickson et al., 2007) and a set of carbon dioxide dissociation constants (Dickson and Millero, 1987). Uncert. = analytical uncertainties of spectrophotometric, potentiometric, and calculated $\mathrm{pH}$. Adjustments of $\mathrm{C}_{\mathrm{T}}, \mathrm{A}_{\mathrm{T}}\left(\right.$ in $\mu \mathrm{mol} \mathrm{kg}{ }^{-1}$ ) and $\mathrm{pH}$ suggested from several a posteriori crossover analysis (Velo et al., 2009, 2010; Pierrot et al., 2010) are also listed.

\begin{tabular}{|c|c|c|c|c|c|c|c|c|c|c|}
\hline \multicolumn{8}{|c|}{ Cruises and $\mathrm{pH}$ measurements } & \multicolumn{3}{|c|}{ Adjustments } \\
\hline Section & Year & P.I. & Expocode & \#Stations & \#Samples & $\mathrm{pH}$ & Uncert. $^{\mathrm{a}}$ & $\mathrm{pH}$ & $\mathrm{C}_{\mathrm{T}}^{\mathrm{b}}$ & $\mathrm{A}_{\mathrm{T}}^{\mathrm{c}}$ \\
\hline TTO & 1981 & T. Takahashi & $316 \mathrm{~N} 19810923$ & 30 & 591 & Calc & 0.008 & 0 & -3.0 & -3.6 \\
\hline BD3 & 1989 & M. Arhan & 35LU19890509 & 20 & 218 & $\mathrm{P}$ & 0.005 & 0.024 & 0 & 0 \\
\hline TYRO & 1990 & G. Fransz & 64TR19900417 & 11 & 189 & Calc & 0.006 & 0 & 0 & 14 \\
\hline AR07E & 1991 & H.M. van Aken & 64TR19910408 & 30 & 616 & Calc & 0.006 & 0 & 6 & 0 \\
\hline $\mathrm{A} 01 \mathrm{E}$ & 1991 & J. Meincke & 06MT18_1 & 26 & 431 & Calc & 0.006 & 0 & 0 & 0 \\
\hline OACES & 1993 & R. Wanninkhof & OACES93 & 28 & 497 & Calc & 0.006 & 0 & 0 & 0 \\
\hline FOUREX & 1997 & S. Bacon & 74DI19970807 & 83 & 1458 & S & 0.004 & -0.005 & 0 & 0 \\
\hline MET97 & 1997 & F. Schott & 06МT19970707 & 8 & 148 & Calc & 0.008 & 0 & 0 & 0 \\
\hline CHAOS & 1998 & Smythe-Wright & 74DI19980423 & 26 & 459 & S & 0.004 & 0.018 & 0 & -8.5 \\
\hline OVIDE'02 & 2002 & H. Mercier & 35 TH20020611 & 85 & 1829 & S & 0.004 & 0 & 0 & 0 \\
\hline $\mathrm{A} 16 \mathrm{~N}$ & 2003 & J. Bullister & 33RO20030604 & 25 & 693 & Calc & 0.006 & 0 & 0 & 0 \\
\hline OVIDE'04 & 2004 & T. Huck & 35 TH20040604 & 98 & 2091 & S & 0.004 & 0 & 0 & 0 \\
\hline OVIDE'06 & 2006 & P. Lherminier & 06M220060523 & 89 & 1937 & $\mathrm{~S}$ & 0.004 & 0 & 0 & 0 \\
\hline OVIDE'08 & 2008 & B. Ferron & $35 \mathrm{TH} 20080610$ & 87 & 2012 & $S$ & 0.004 & 0 & 0 & 0 \\
\hline
\end{tabular}

\footnotetext{
a The uncertainty of \pm 0.006 ( \pm 0.008 for TTO) estimated for the calculated pHs (Calc) was obtained by random propagation of the reported uncertainties of $\mathrm{C}_{\mathrm{T}}$ and $\mathrm{A}_{\mathrm{T}}$ in ${ }^{\mathrm{b}}$ and ${ }^{c}$ below. ${ }^{b} C_{T}$ analysed with SOMMA (Johnson et al., 1993) and calibrated with CRMs given analytical accuracy $\pm 2 \mu$ mol kg ${ }^{-1}$, except in 1981 TTO cruise that was determined potentiometrically (Bradshaw et al., 1981) without CRMs with accuracy $\pm 4 \mu \mathrm{mol} \mathrm{kg}{ }^{-1} .{ }^{c} \mathrm{~A}_{\mathrm{T}}$ analysed with potentiometric titration and determined by developing either a full titration curve (Millero et al., 1993; Dickson et al., 2007) or from single point titration (Pérez and Fraga, 1987; Mintrop et al., 2002). Analytical accuracy $\pm 4 \mu \mathrm{mol} \mathrm{kg}^{-1}$.
}

scale as specified in Pérez and Fraga (1987). Some of the cruises listed in Table 1 did not perform direct $\mathrm{pH}$ measurements, but obtained total alkalinity $\left(\mathrm{A}_{\mathrm{T}}\right)$ and dissolved inorganic carbon $\left(\mathrm{C}_{\mathrm{T}}\right)$ data instead. In such cases the $\mathrm{pH}$ values were calculated in the SWS scale from $\mathrm{A}_{\mathrm{T}}$ and $\mathrm{C}_{\mathrm{T}}$ data using the thermodynamic equations of the carbon system (Dickson et al., 2007) and a set of $\mathrm{CO}_{2}$ dissociation constants (Dickson and Millero, 1987). An uncertainty of \pm 0.006 was estimated for these calculated $\mathrm{pHs}$ by random propagation of the reported $\mathrm{A}_{\mathrm{T}}$ and $\mathrm{C}_{\mathrm{T}}$ uncertainties $\left( \pm 4\right.$ and $\pm 2 \mu \mathrm{mol} \mathrm{kg}{ }^{-1}$, respectively). The exception to the latter is the TTO cruise, which had a reported $\mathrm{C}_{\mathrm{T}}$ uncertainty of $\pm 4 \mu \mathrm{mol} \mathrm{kg}{ }^{-1}$ that caused the estimated uncertainty for calculated $\mathrm{pH}$ to be slightly higher $( \pm 0.008$; Table 1$)$.

During the A16N cruise $\mathrm{pH}$ was determined spectrophotometrically, but the spatial resolution was not as good as in the case of $\mathrm{C}_{\mathrm{T}}$ and $\mathrm{A}_{\mathrm{T}}$, so we used $\mathrm{pH}$ values calculated from $\mathrm{C}_{\mathrm{T}}$ and $\mathrm{A}_{T}$ for this cruise instead. The AR7E and A01E cruises (Fig. 1a) had a comprehensive amount of $\mathrm{C}_{\mathrm{T}}$ measurements, yet very little potentiometric $A_{T}$ data. Given the scarcity of $\mathrm{A}_{\mathrm{T}}$ data, the equation $\mathrm{A}_{\mathrm{T}}=S / 35 \cdot\left(2294.7+1.37\left[\mathrm{Si}(\mathrm{OH})_{4}\right]\right)$ $\left(R^{2}=0.97 ;\left[\mathrm{Si}(\mathrm{OH})_{4}\right]\right.$ refers to silicate concentration) given by Pérez et al. (2010) was applied to the AR7E and A01E datasets to generate $A_{T}$ values at the sampling depths of measured $\mathrm{C}_{\mathrm{T}}$. The $\mathrm{pH}$ was then calculated from $\mathrm{C}_{\mathrm{T}}$ and $\mathrm{A}_{\mathrm{T}}$ data as mentioned above.

\section{$2.2 \mathrm{pH}$ data analysis}

The dataset spans 27 yr (1981-2008) and a wide spatial coverage of the study area (Fig. 1a; Table 1) that was divided into three basins: the Irminger, Iceland and East North Atlantic (ENA). These three basins and their geographical boundaries have also been defined and used in Pérez et al. (2010). The Irminger Basin boundaries are defined by the main axis of the Reykjanes Ridge and the east coast of Greenland. The Iceland Basin is defined as the region bounded between the main axis of the Reykjanes Ridge and the line joining the Eriador Seamount and the Faroe Islands. The ENA Basin extends south from the Eriador-Faroe Line over the Rockall Trough, the Porcupine Bank, and the Biscay and Iberian Basins (Fig. 1).

In order to evaluate the temporal variability of $\mathrm{pH}$ in the water masses of the North Atlantic, the water column was divided into five layers delimited by potential density ( $\sigma_{\theta}$ isopycnals) for each region (Fig. 1b). To determine the isopycnal boundaries of the North Atlantic Deep Water (NADW) we followed Lherminier et al. (2010), who established different layers by potential density intervals on the basis of the hydrographic properties and circulation of the different water masses along the OVIDE section. They discriminate between the two components of NADW: the lower NADW (INADW), spreading from the bottom to $\sigma_{\theta}=$ 45.84 , and the upper NADW (uNADW) that spreads in 
the density range $36.94<\sigma_{\theta}<45.84$. We took the density range $37<\sigma_{\theta}<45.84$, which is almost identical, because the isopycnal $\sigma_{\theta}=37$ seemed to delimit better the deepest boundary of the cLSW core (coincident with the uNADW upper density limit) in the Iceland and ENA Basins. For the spreading of LSW in the ENA Basin, the density range selected $\left(32.35<\sigma_{\theta}<37\right)$ is very close to the one in Lherminier et al. (2010). Following Ríos et al. (1992) the Mediterranean Water (MW) layer is delimited by $27.2<\sigma_{\theta}<32.35$ and the North Atlantic Central Water (NACW) layer is established from surface to $\sigma_{\theta}<32.35$, according to the spreading of these water masses in the zone. For the Irminger and Iceland Basins, the potential density limits were established following Kieke et al. (2007) and Yashayaev et al. (2008). So for the Iceland Basin the layer of SPMW is found between $100 \mathrm{~m}$ and $\sigma_{\theta}=27.6$. The upper and classical LSW (uLSW and cLSW) spread in the density ranges of $27.68<\sigma_{\theta}<27.76$ and $27.76<\sigma_{\theta}<27.81$, respectively. In the Irminger Basin, the Sub Arctic Intermediate Water (SAIW) spreads between $100 \mathrm{~m}$ and $\sigma_{\theta}=27.68$. The uLSW and cLSW are found between $27.68<\sigma_{\theta}<27.76$ and $27.76<\sigma_{\theta}<27.81$, respectively. The North Atlantic Deep Water (NADW, which includes contributions from ISOW) is delimited by $27.81<\sigma_{\theta}<27.88$, and the Denmark Strait Overflow Water (DSOW) by $\sigma_{\theta}>27.88$ (Fig. 1b).

\subsubsection{Basin normalization of average $\mathrm{pH}_{\mathrm{SWS25}}$}

The average $\mathrm{pH}_{\mathrm{SWS} 25}$ was obtained for each layer and year in the three basins, following the averaging and "basinreferencing" methodology that Pérez et al. $(2008,2010)$ and Ríos et al. (2012) used for $C_{a n t}$. The spatial coverage of cruises over years is variable and this can cause significant biases in the observed average layer properties in each year. These small differences can potentially introduce spatial biases in the average $\mathrm{pH}_{\mathrm{SwS} 25}$ due to different ventilation stages and rates of each water mass. Therefore, for each basin the $\mathrm{pH}_{\mathrm{SWS} 25}$ was normalized to better represent the $\mathrm{pH}_{\mathrm{SWS} 25}$ in each considered layer of the basin (Fig. 1) by adding a new term named $\Delta \mathrm{pH}_{\mathrm{SWS} 25-\mathrm{BA}}$ (where "BA" stands for basin average). This term represents the deviation of $\mathrm{pH}_{\text {SWS25 }}$ (average from cruise data) from the $\mathrm{pH}_{\mathrm{SWS} 25}$ basin average $\left(\mathrm{pH}_{\mathrm{SWS} 25-\mathrm{BA}}\right)$.

$\mathrm{pH}_{\mathrm{SWS} 25-\mathrm{BA}}=\mathrm{pH}_{\mathrm{SWS} 25}+\Delta \mathrm{pH}_{\mathrm{SWS} 25-\mathrm{BA}}$

The $\Delta \mathrm{pH}_{\mathrm{SWS} 25-\mathrm{BA}}$ term was computed from cruise data and expressed as individual correction elements for each cruise and layer in the three basins (Table 2) as follows:

$\Delta \mathrm{pH}_{\mathrm{SWS25-BA}}=\sum_{i=1}^{4} a_{i}\left(X_{i}^{\mathrm{WOA} 05}-X_{i}^{c}\right)$,

where $c$ stands for "cruise" and subscript $i$ denotes "property" $\left(1=\mathrm{Si}(\mathrm{OH})_{4} ; 2=\mathrm{AOU} ; 3=\theta ; 4=S\right)$. The $X_{i}^{c}$ and $X_{i}^{\mathrm{WOA} 05}$ terms are, respectively, the average magnitudes of the $i$ th properties calculated from direct observations along the cruise track and from WOA05 data in the respective basins (Table 2). The $a_{i}$ factors are the regression coefficients that were calculated in each basin and layer from a multiple linear regression (MLR) fit (Eq. 3) of the $\mathrm{pH}_{\mathrm{SWS} 25}$ averages vs. the averaged $i$ properties using data from the fourteen cruises (Table 2). The obtained $a_{i}$ regression coefficients are listed in Table 3.

$\mathrm{pH}_{\mathrm{SWS} 25}^{\mathrm{MLR}}=\sum_{i=1}^{5} a_{i} X_{i}^{c}+k$

All terms and scripts in Eq. (3) have the same meaning as in Eq. (2). Also, the $X_{i}^{c}$ terms for $i=1$ through 4 are the same as in Eq. (2). The same is true for the $a_{i}$ coefficients in equation (2). Actually, the purpose of Eq. (3) is obtaining those $a_{i}$ values to be used in Eq. (2). $k$ are the independent terms. The $X_{5}=x \mathrm{CO}_{2}^{\mathrm{atm}}$ values used as input parameters in Eq. (3) are the averages for the year of the corresponding cruise $c$. The $x \mathrm{CO}_{2}^{\text {atm }}$ records were obtained from time series from meteorological stations in the NASPG (Storhofdi, Iceland; CIBA, Spain; Mace Head, Ireland; Ocean Station C, US; Pico-Azores, Portugal; and Terceira Island-Azores, Portugal), that are part of the global cooperative air-sampling network managed and operated by the National Oceanic and Atmospheric Administration (NOAA) Carbon Cycle Greenhouse Gas group (http://www.esrl.noaa.gov/gmd/ccgg/flask. $\mathrm{html}$ ). The $a_{5}$ term associated with the $x \mathrm{CO}_{2}^{\mathrm{atm}}$ variable (Table 3) in Eq. (3) is not used in Eq. (2) since the $\Delta \mathrm{pH}_{\mathrm{SWS} 25-\mathrm{BA}}$ term should only include the effect of variables with spatial variation. Such $x \mathrm{CO}_{2}^{\text {atm }}$ terms are required when calculating the $a_{i}$ coefficients (Eq. 3, Table 3), since $x \mathrm{CO}_{2}^{\text {atm }}$ co-varies

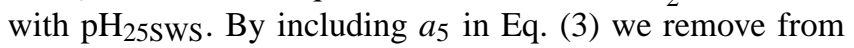
the rest of $a_{i}$ factors the transient influences that co-vary with $\mathrm{pH}_{\mathrm{SWS25}}$. Considering that $\mathrm{pH}$ varies over time because of the $x \mathrm{CO}_{2}^{\text {atm }}$ change, the inclusion of this variable in Eq. (3) insures that coefficients of the other properties that change mostly spatially are more consistent than if the $x \mathrm{CO}_{2}^{\text {atm }}$ is not included.

\section{Results}

The vertical distributions of $\mathrm{pH}_{\mathrm{SWS} 25}$ along a section between the Iberian Peninsula and Greenland are shown in Fig. 2, providing a first look at the evolution of $\mathrm{pH}$ over the last two decades. The general pattern of $\mathrm{pH}_{\mathrm{SWS} 25}$ follows the natural distribution expected, with higher $\mathrm{pH}$ values at the surface and lower $\mathrm{pH}$ in deep waters: the high values of $\mathrm{pH}_{\mathrm{SWS} 25}$ above the seasonal thermocline, in the photic layer (uppermost $\sim 400 \mathrm{~m}$ ), respond to the photosynthetic activity of primary producers that withdraw dissolved $\mathrm{CO}_{2}$ from seawater. The deep and less ventilated NADW has low $\mathrm{pH}_{\text {SWS25. The NADW is located generally below } 2500 \mathrm{dbar}}$ $\left(\sigma_{2}>37.00\right.$; Fig. 1b) mainly in the deep ENA Basin and shows weak signs of acidification over the last two decades, 
Table 2a. Temporal evolution (1981-2008) of the values (average \pm standard error of the mean) of salinity, potential temperature, AOU, silicate concentrations, measured $\mathrm{pH}\left(\mathrm{pH}_{\mathrm{SWS} 25}\right), \mathrm{pH}$ basin corrections $\left(\Delta_{\mathrm{pH}} \mathrm{SWS}_{\mathrm{S} 25-\mathrm{BA}}\right)$ and basin-normalized $\mathrm{pH}\left(\mathrm{pH}_{\mathrm{SWS} 25-\mathrm{BA}}=\right.$ $\mathrm{pH}_{\mathrm{SWS} 25}+\Delta \mathrm{pH}_{\mathrm{SWS} 25-\mathrm{BA}}$ ) for the water masses considered in the: (a) Irminger; (b) Iceland; and (c) ENA Basins. The WOA05 lines give the climatological data used as reference values (see Eq. 2). Irminger Basin.

\begin{tabular}{|c|c|c|c|c|c|c|c|}
\hline Year & Salinity & $\begin{array}{l}\theta \\
\left({ }^{\circ} \mathrm{C}\right)\end{array}$ & $\begin{array}{l}\text { AOU } \\
\left(\mu \mathrm{mol} \mathrm{kg}{ }^{-1}\right)\end{array}$ & $\begin{array}{l}\mathrm{Si}(\mathrm{OH})_{4} \\
\left(\mu \mathrm{mol} \mathrm{kg}^{-1}\right)\end{array}$ & $\mathrm{pH}_{\mathrm{SWS} 25}$ & $\Delta \mathrm{pH}_{\mathrm{SWS} 25-\mathrm{BA}}$ & $\mathrm{pH}_{\mathrm{SWS} 25-\mathrm{BA}}$ \\
\hline \multicolumn{8}{|c|}{ SAIW $\left(\sigma_{0}<27.68 \mathrm{~kg} \mathrm{~m}^{-3}\right)$} \\
\hline 1981 & $34.894 \pm 0.002$ & $5.370 \pm 0.006$ & $18.6 \pm 0.3$ & $7.96 \pm 0.09$ & $7.776 \pm 0.002$ & $0.014 \pm 0.003$ & $7.790 \pm 0.003$ \\
\hline 1991 & $34.981 \pm 0.002$ & $5.228 \pm 0.010$ & $19.0 \pm 0.5$ & $8.50 \pm 0.15$ & $7.768 \pm 0.002$ & $0.006 \pm 0.003$ & $7.774 \pm 0.004$ \\
\hline 1991 & $34.962 \pm 0.001$ & $5.482 \pm 0.004$ & $25.8 \pm 0.2$ & $8.96 \pm 0.05$ & $7.756 \pm 0.001$ & $0.016 \pm 0.003$ & $7.772 \pm 0.003$ \\
\hline 1997 & $34.911 \pm 0.001$ & $5.902 \pm 0.005$ & $27.9 \pm 0.2$ & $7.69 \pm 0.07$ & $7.758 \pm 0.001$ & $0.007 \pm 0.003$ & $7.764 \pm 0.003$ \\
\hline 1997 & $34.893 \pm 0.001$ & $5.134 \pm 0.003$ & $30.1 \pm 0.2$ & $8.83 \pm 0.05$ & $7.736 \pm 0.001$ & $0.027 \pm 0.003$ & $7.763 \pm 0.003$ \\
\hline 2002 & $34.949 \pm 0.001$ & $5.362 \pm 0.004$ & $24.6 \pm 0.2$ & $8.08 \pm 0.06$ & $7.747 \pm 0.001$ & $0.006 \pm 0.003$ & $7.753 \pm 0.003$ \\
\hline 2004 & $34.967 \pm 0.001$ & $5.611 \pm 0.003$ & $23.8 \pm 0.1$ & $7.93 \pm 0.04$ & $7.746 \pm 0.001$ & $0.000 \pm 0.003$ & $7.746 \pm 0.003$ \\
\hline 2006 & $34.977 \pm 0.001$ & $5.660 \pm 0.003$ & $24.1 \pm 0.1$ & $7.88 \pm 0.04$ & $7.745 \pm 0.001$ & $-0.003 \pm 0.003$ & $7.742 \pm 0.003$ \\
\hline 2008 & $34.978 \pm 0.001$ & $5.926 \pm 0.002$ & $12.4 \pm 0.1$ & $7.17 \pm 0.04$ & $7.757 \pm 0.001$ & $-0.013 \pm 0.003$ & $7.744 \pm 0.003$ \\
\hline WOA05 & $34.964 \pm 0.004$ & $5.544 \pm 0.027$ & $19.7 \pm 0.3$ & $7.89 \pm 0.04$ & $*$ & $*$ & $*$ \\
\hline \multicolumn{8}{|c|}{$\operatorname{uLSW}\left(27.68<\sigma_{0}<27.76 \mathrm{~kg} \mathrm{~m}^{-3}\right)$} \\
\hline 1981 & $34.865 \pm 0.001$ & $3.534 \pm 0.004$ & $28.1 \pm 0.2$ & $9.28 \pm 0.07$ & $7.749 \pm 0.001$ & $0.022 \pm 0.002$ & $7.771 \pm 0.002$ \\
\hline 1991 & $34.889 \pm 0.001$ & $3.577 \pm 0.004$ & $24.2 \pm 0.2$ & $9.52 \pm 0.07$ & $7.745 \pm 0.001$ & $0.010 \pm 0.002$ & $7.755 \pm 0.002$ \\
\hline 1991 & $34.900 \pm 0.001$ & $3.728 \pm 0.003$ & $28.7 \pm 0.2$ & $10.05 \pm 0.05$ & $7.740 \pm 0.001$ & $0.013 \pm 0.002$ & $7.753 \pm 0.002$ \\
\hline 1997 & $34.877 \pm 0.001$ & $3.533 \pm 0.005$ & $35.6 \pm 0.3$ & $9.67 \pm 0.08$ & $7.725 \pm 0.001$ & $0.017 \pm 0.002$ & $7.742 \pm 0.002$ \\
\hline 1997 & $34.869 \pm 0.001$ & $3.520 \pm 0.003$ & $35.9 \pm 0.1$ & $10.17 \pm 0.04$ & $7.716 \pm 0.001$ & $0.029 \pm 0.002$ & $7.746 \pm 0.002$ \\
\hline 2002 & $34.896 \pm 0.001$ & $3.803 \pm 0.003$ & $35.0 \pm 0.1$ & $9.30 \pm 0.04$ & $7.725 \pm 0.001$ & $0.010 \pm 0.002$ & $7.735 \pm 0.002$ \\
\hline 2004 & $34.888 \pm 0.001$ & $3.710 \pm 0.003$ & $37.2 \pm 0.1$ & $9.50 \pm 0.04$ & $7.719 \pm 0.001$ & $0.014 \pm 0.002$ & $7.733 \pm 0.002$ \\
\hline 2006 & $34.902 \pm 0.001$ & $3.831 \pm 0.002$ & $34.4 \pm 0.1$ & $9.48 \pm 0.04$ & $7.720 \pm 0.001$ & $0.008 \pm 0.002$ & $7.728 \pm 0.002$ \\
\hline 2008 & $34.908 \pm 0.001$ & $3.913 \pm 0.002$ & $27.2 \pm 0.1$ & $9.21 \pm 0.03$ & $7.721 \pm 0.001$ & $0.004 \pm 0.002$ & $7.725 \pm 0.002$ \\
\hline WOA05 & $34.925 \pm 0.002$ & $3.997 \pm 0.020$ & $33.5 \pm 0.2$ & $9.62 \pm 0.04$ & $*$ & $*$ & $*$ \\
\hline \multicolumn{8}{|c|}{$\operatorname{cLSW}\left(27.6<\sigma_{0}<27.81 \mathrm{~kg} \mathrm{~m}^{-3}\right)$} \\
\hline 1981 & $34.917 \pm 0.002$ & $3.375 \pm 0.008$ & $39.1 \pm 0.4$ & $10.58 \pm 0.12$ & $7.749 \pm 0.002$ & $0.008 \pm 0.002$ & $7.757 \pm 0.003$ \\
\hline 1991 & $34.879 \pm 0.001$ & $3.137 \pm 0.003$ & $32.6 \pm 0.2$ & $10.16 \pm 0.05$ & $7.742 \pm 0.001$ & $0.007 \pm 0.002$ & $7.749 \pm 0.002$ \\
\hline 1991 & $34.881 \pm 0.001$ & $3.156 \pm 0.003$ & $29.8 \pm 0.1$ & $10.30 \pm 0.04$ & $7.738 \pm 0.001$ & $0.009 \pm 0.002$ & $7.747 \pm 0.002$ \\
\hline 1997 & $34.871 \pm 0.001$ & $2.986 \pm 0.004$ & $31.1 \pm 0.2$ & $9.94 \pm 0.06$ & $7.729 \pm 0.001$ & $0.015 \pm 0.002$ & $7.744 \pm 0.002$ \\
\hline 1997 & $34.868 \pm 0.001$ & $2.989 \pm 0.003$ & $30.9 \pm 0.1$ & $10.43 \pm 0.04$ & $7.722 \pm 0.001$ & $0.022 \pm 0.002$ & $7.743 \pm 0.002$ \\
\hline 2002 & $34.897 \pm 0.001$ & $3.184 \pm 0.003$ & $38.8 \pm 0.2$ & $10.23 \pm 0.05$ & $7.727 \pm 0.001$ & $0.011 \pm 0.002$ & $7.738 \pm 0.002$ \\
\hline 2004 & $34.902 \pm 0.001$ & $3.232 \pm 0.004$ & $40.5 \pm 0.2$ & $10.44 \pm 0.06$ & $7.722 \pm 0.001$ & $0.013 \pm 0.002$ & $7.735 \pm 0.002$ \\
\hline 2006 & $34.923 \pm 0.001$ & $3.369 \pm 0.003$ & $40.8 \pm 0.2$ & $10.58 \pm 0.05$ & $7.723 \pm 0.001$ & $0.011 \pm 0.002$ & $7.734 \pm 0.002$ \\
\hline 2008 & $34.924 \pm 0.001$ & $3.383 \pm 0.003$ & $39.5 \pm 0.2$ & $10.72 \pm 0.05$ & $7.722 \pm 0.001$ & $0.013 \pm 0.002$ & $7.734 \pm 0.002$ \\
\hline WOA05 & $34.899 \pm 0.001$ & $3.314 \pm 0.012$ & $35.7 \pm 0.1$ & $10.25 \pm 0.03$ & $*$ & $*$ & $*$ \\
\hline \multicolumn{8}{|c|}{ uNADW $\left(27.81<\sigma_{0}<27.88 \mathrm{~kg} \mathrm{~m}^{-3}\right)$} \\
\hline 1981 & $34.948 \pm 0.001$ & $2.980 \pm 0.005$ & $44.3 \pm 0.2$ & $12.01 \pm 0.07$ & $7.750 \pm 0.001$ & $0.000 \pm 0.003$ & $7.750 \pm 0.003$ \\
\hline 1991 & $34.940 \pm 0.001$ & $2.925 \pm 0.003$ & $48.4 \pm 0.2$ & $12.66 \pm 0.05$ & $7.745 \pm 0.001$ & $0.000 \pm 0.003$ & $7.745 \pm 0.003$ \\
\hline 1991 & $34.935 \pm 0.001$ & $2.887 \pm 0.003$ & $44.9 \pm 0.2$ & $12.79 \pm 0.05$ & $7.745 \pm 0.001$ & $0.000 \pm 0.003$ & $7.745 \pm 0.003$ \\
\hline 1997 & $34.917 \pm 0.001$ & $2.785 \pm 0.005$ & $41.5 \pm 0.3$ & $11.26 \pm 0.08$ & $7.740 \pm 0.001$ & $0.000 \pm 0.003$ & $7.740 \pm 0.003$ \\
\hline 1997 & $34.924 \pm 0.001$ & $2.813 \pm 0.004$ & $44.2 \pm 0.2$ & $12.81 \pm 0.06$ & $7.733 \pm 0.001$ & $0.000 \pm 0.003$ & $7.733 \pm 0.003$ \\
\hline 2002 & $34.918 \pm 0.001$ & $2.759 \pm 0.003$ & $43.7 \pm 0.1$ & $11.50 \pm 0.04$ & $7.732 \pm 0.001$ & $0.000 \pm 0.003$ & $7.732 \pm 0.003$ \\
\hline 2004 & $34.916 \pm 0.001$ & $2.753 \pm 0.003$ & $44.4 \pm 0.1$ & $11.70 \pm 0.04$ & $7.726 \pm 0.001$ & $0.000 \pm 0.003$ & $7.726 \pm 0.003$ \\
\hline 2006 & $34.930 \pm 0.001$ & $2.859 \pm 0.003$ & $43.2 \pm 0.1$ & $11.70 \pm 0.04$ & $7.728 \pm 0.001$ & $0.000 \pm 0.003$ & $7.728 \pm 0.003$ \\
\hline 2008 & $34.931 \pm 0.001$ & $2.872 \pm 0.003$ & $41.4 \pm 0.2$ & $11.96 \pm 0.05$ & $7.726 \pm 0.001$ & $0.000 \pm 0.003$ & $7.726 \pm 0.003$ \\
\hline WOA05 & $34.915 \pm 0.003$ & $2.869 \pm 0.028$ & $42.7 \pm 0.2$ & $11.30 \pm 0.10$ & $*$ & $*$ & $*$ \\
\hline \multicolumn{8}{|c|}{ DSOW $\left(\sigma_{0}>27.88 \mathrm{~kg} \mathrm{~m}^{-3}\right)$} \\
\hline 1981 & $34.892 \pm 0.002$ & $1.679 \pm 0.008$ & $36.7 \pm 0.4$ & $9.80 \pm 0.12$ & $7.749 \pm 0.002$ & $0.000 \pm 0.005$ & $7.749 \pm 0.006$ \\
\hline 1991 & $34.897 \pm 0.001$ & $1.778 \pm 0.005$ & $41.6 \pm 0.3$ & $10.20 \pm 0.08$ & $7.742 \pm 0.001$ & $0.000 \pm 0.005$ & $7.742 \pm 0.005$ \\
\hline 1991 & $34.896 \pm 0.001$ & $1.794 \pm 0.006$ & $38.1 \pm 0.3$ & $10.57 \pm 0.09$ & $7.742 \pm 0.001$ & $0.000 \pm 0.005$ & $7.742 \pm 0.005$ \\
\hline 1997 & $34.897 \pm 0.002$ & $1.772 \pm 0.009$ & $38.0 \pm 0.5$ & $9.73 \pm 0.14$ & $7.726 \pm 0.002$ & $0.000 \pm 0.005$ & $7.726 \pm 0.006$ \\
\hline 1997 & $34.894 \pm 0.002$ & $1.720 \pm 0.008$ & $38.8 \pm 0.4$ & $11.11 \pm 0.13$ & $7.724 \pm 0.002$ & $0.000 \pm 0.005$ & $7.724 \pm 0.006$ \\
\hline 2002 & $34.887 \pm 0.001$ & $1.721 \pm 0.005$ & $39.4 \pm 0.3$ & $9.53 \pm 0.08$ & $7.731 \pm 0.001$ & $0.000 \pm 0.005$ & $7.731 \pm 0.005$ \\
\hline 2004 & $34.869 \pm 0.001$ & $1.535 \pm 0.005$ & $36.3 \pm 0.2$ & $8.92 \pm 0.07$ & $7.723 \pm 0.001$ & $0.000 \pm 0.005$ & $7.723 \pm 0.005$ \\
\hline 2006 & $34.906 \pm 0.001$ & $1.874 \pm 0.004$ & $37.7 \pm 0.2$ & $9.77 \pm 0.07$ & $7.726 \pm 0.001$ & $0.000 \pm 0.005$ & $7.726 \pm 0.005$ \\
\hline 2008 & $34.908 \pm 0.001$ & $1.797 \pm 0.006$ & $36.4 \pm 0.3$ & $10.06 \pm 0.09$ & $7.721 \pm 0.001$ & $0.000 \pm 0.005$ & $7.721 \pm 0.005$ \\
\hline WOA05 & $34.895 \pm 0.004$ & $1.885 \pm 0.064$ & $40.1 \pm 0.2$ & $11.08 \pm 0.19$ & $*$ & $*$ & $*$ \\
\hline
\end{tabular}


Table 2b. Iceland Basin.

\begin{tabular}{|c|c|c|c|c|c|c|c|}
\hline Year & Salinity & $\begin{array}{l}\theta \\
\left({ }^{\circ} \mathrm{C}\right)\end{array}$ & $\begin{array}{l}\mathrm{AOU} \\
\left(\mu \mathrm{mol} \mathrm{kg}{ }^{-1}\right)\end{array}$ & $\begin{array}{l}\mathrm{Si}(\mathrm{OH})_{4} \\
\left(\mu \mathrm{mol} \mathrm{kg}{ }^{-1}\right)\end{array}$ & $\mathrm{pH}_{\mathrm{SWS} 25}$ & $\Delta \mathrm{pH}_{\mathrm{SWS} 25-\mathrm{BA}}$ & $\mathrm{pH}_{\mathrm{SWS} 25-\mathrm{BA}}$ \\
\hline \multicolumn{8}{|c|}{$\operatorname{SPMW}\left(\sigma_{0}<27.60 \mathrm{~kg} \mathrm{~m}^{-3}\right)$} \\
\hline 1981 & $35.183 \pm 0.001$ & $8.242 \pm 0.003$ & $28.2 \pm 0.1$ & $6.89 \pm 0.04$ & $7.814 \pm 0.001$ & $-0.008 \pm 0.005$ & $7.806 \pm 0.005$ \\
\hline 1991 & $35.109 \pm 0.001$ & $7.030 \pm 0.003$ & $22.9 \pm 0.2$ & $7.73 \pm 0.05$ & $7.797 \pm 0.001$ & $0.005 \pm 0.005$ & $7.802 \pm 0.005$ \\
\hline 1991 & $35.000 \pm 0.002$ & $6.935 \pm 0.007$ & $37.8 \pm 0.3$ & $8.94 \pm 0.10$ & $7.757 \pm 0.001$ & $0.030 \pm 0.005$ & $7.787 \pm 0.005$ \\
\hline 1993 & $35.151 \pm 0.001$ & $7.889 \pm 0.003$ & $31.6 \pm 0.1$ & $6.68 \pm 0.04$ & $7.789 \pm 0.001$ & $0.004 \pm 0.005$ & $7.793 \pm 0.005$ \\
\hline 1997 & $35.094 \pm 0.001$ & $7.931 \pm 0.003$ & $47.2 \pm 0.2$ & $8.17 \pm 0.05$ & $7.769 \pm 0.001$ & $0.028 \pm 0.005$ & $7.797 \pm 0.005$ \\
\hline 1998 & $35.249 \pm 0.001$ & $8.563 \pm 0.003$ & $28.7 \pm 0.2$ & $7.22 \pm 0.05$ & $7.796 \pm 0.001$ & $-0.011 \pm 0.005$ & $7.785 \pm 0.005$ \\
\hline 2002 & $35.117 \pm 0.001$ & $7.614 \pm 0.003$ & $30.5 \pm 0.2$ & $7.33 \pm 0.05$ & $7.776 \pm 0.001$ & $0.008 \pm 0.005$ & $7.784 \pm 0.005$ \\
\hline 2003 & $35.260 \pm 0.001$ & $8.826 \pm 0.002$ & $37.4 \pm 0.1$ & $6.68 \pm 0.03$ & $7.785 \pm 0.001$ & $-0.002 \pm 0.005$ & $7.783 \pm 0.005$ \\
\hline 2004 & $35.105 \pm 0.001$ & $7.719 \pm 0.002$ & $38.5 \pm 0.1$ & $7.40 \pm 0.04$ & $7.763 \pm 0.001$ & $0.018 \pm 0.005$ & $7.782 \pm 0.005$ \\
\hline 2006 & $35.077 \pm 0.001$ & $7.906 \pm 0.003$ & $38.7 \pm 0.1$ & $7.20 \pm 0.04$ & $7.765 \pm 0.001$ & $0.016 \pm 0.005$ & $7.781 \pm 0.005$ \\
\hline 2008 & $35.111 \pm 0.001$ & $7.809 \pm 0.002$ & $28.1 \pm 0.1$ & $6.84 \pm 0.04$ & $7.771 \pm 0.001$ & $0.000 \pm 0.005$ & $7.771 \pm 0.005$ \\
\hline WOA05 & $35.175 \pm 0.002$ & $7.954 \pm 0.027$ & $29.6 \pm 0.4$ & $7.36 \pm 0.04$ & $*$ & $*$ & $*$ \\
\hline \multicolumn{8}{|c|}{$\mathrm{uLSW}\left(\sigma_{0}>27.60 \mathrm{~kg} \mathrm{~m}^{-3} ; \sigma_{1}<32.35 \mathrm{~kg} \mathrm{~m}^{-3}\right)$} \\
\hline 1981 & $34.982 \pm 0.001$ & $4.637 \pm 0.005$ & $48.5 \pm 0.3$ & $10.56 \pm 0.08$ & $7.739 \pm 0.001$ & $-0.002 \pm 0.002$ & $7.737 \pm 0.002$ \\
\hline 1991 & $34.959 \pm 0.001$ & $4.488 \pm 0.004$ & $56.2 \pm 0.2$ & $10.87 \pm 0.06$ & $7.726 \pm 0.001$ & $0.000 \pm 0.002$ & $7.727 \pm 0.002$ \\
\hline 1991 & $34.944 \pm 0.001$ & $4.340 \pm 0.006$ & $51.7 \pm 0.3$ & $11.16 \pm 0.09$ & $7.728 \pm 0.001$ & $0.002 \pm 0.002$ & $7.729 \pm 0.002$ \\
\hline 1993 & $34.987 \pm 0.001$ & $4.754 \pm 0.004$ & $56.0 \pm 0.2$ & $10.43 \pm 0.06$ & $7.730 \pm 0.001$ & $-0.003 \pm 0.002$ & $7.727 \pm 0.002$ \\
\hline 1997 & $34.940 \pm 0.001$ & $4.345 \pm 0.003$ & $55.7 \pm 0.2$ & $11.11 \pm 0.05$ & $7.715 \pm 0.001$ & $0.002 \pm 0.002$ & $7.717 \pm 0.002$ \\
\hline 1998 & $35.009 \pm 0.002$ & $4.938 \pm 0.007$ & $62.2 \pm 0.3$ & $11.72 \pm 0.10$ & $7.712 \pm 0.001$ & $0.006 \pm 0.002$ & $7.718 \pm 0.002$ \\
\hline 2002 & $34.976 \pm 0.001$ & $4.657 \pm 0.003$ & $57.5 \pm 0.2$ & $10.60 \pm 0.05$ & $7.716 \pm 0.001$ & $-0.002 \pm 0.002$ & $7.714 \pm 0.002$ \\
\hline 2003 & $35.024 \pm 0.001$ & $4.979 \pm 0.004$ & $63.7 \pm 0.2$ & $11.24 \pm 0.06$ & $7.709 \pm 0.001$ & $0.003 \pm 0.002$ & $7.712 \pm 0.002$ \\
\hline 2004 & $34.948 \pm 0.001$ & $4.408 \pm 0.002$ & $55.7 \pm 0.1$ & $10.68 \pm 0.04$ & $7.709 \pm 0.001$ & $0.000 \pm 0.002$ & $7.708 \pm 0.002$ \\
\hline 2006 & $34.956 \pm 0.001$ & $4.494 \pm 0.003$ & $54.4 \pm 0.1$ & $10.74 \pm 0.04$ & $7.707 \pm 0.001$ & $-0.001 \pm 0.002$ & $7.706 \pm 0.002$ \\
\hline 2008 & $34.952 \pm 0.001$ & $4.451 \pm 0.003$ & $51.1 \pm 0.1$ & $10.50 \pm 0.04$ & $7.709 \pm 0.001$ & $-0.002 \pm 0.002$ & $7.707 \pm 0.002$ \\
\hline WOA05 & $35.015 \pm 0.002$ & $4.924 \pm 0.001$ & $54.1 \pm 0.3$ & $10.79 \pm 0.05$ & $*$ & $*$ & $*$ \\
\hline \multicolumn{8}{|c|}{$\operatorname{cLSW}\left(\sigma_{1}>32.35 \mathrm{~kg} \mathrm{~m}^{-3} ; \sigma_{2}<37.00 \mathrm{~kg} \mathrm{~m}^{-3}\right)$} \\
\hline 1981 & $34.943 \pm 0.001$ & $3.517 \pm 0.004$ & $44.7 \pm 0.2$ & $12.13 \pm 0.07$ & $7.738 \pm 0.001$ & $0.009 \pm 0.006$ & $7.747 \pm 0.006$ \\
\hline 1991 & $34.928 \pm 0.001$ & $3.376 \pm 0.003$ & $45.6 \pm 0.1$ & $11.59 \pm 0.04$ & $7.746 \pm 0.001$ & $-0.002 \pm 0.006$ & $7.743 \pm 0.006$ \\
\hline 1991 & $34.923 \pm 0.001$ & $3.308 \pm 0.005$ & $43.5 \pm 0.3$ & $12.31 \pm 0.08$ & $7.740 \pm 0.001$ & $0.002 \pm 0.006$ & $7.742 \pm 0.006$ \\
\hline 1993 & $34.926 \pm 0.001$ & $3.458 \pm 0.005$ & $44.6 \pm 0.2$ & $11.27 \pm 0.07$ & $7.737 \pm 0.001$ & $-0.003 \pm 0.006$ & $7.735 \pm 0.006$ \\
\hline 1997 & $34.905 \pm 0.001$ & $3.170 \pm 0.002$ & $40.1 \pm 0.1$ & $11.72 \pm 0.04$ & $7.732 \pm 0.001$ & $-0.009 \pm 0.006$ & $7.723 \pm 0.006$ \\
\hline 1998 & $34.919 \pm 0.002$ & $3.372 \pm 0.006$ & $44.1 \pm 0.3$ & $12.08 \pm 0.09$ & $7.718 \pm 0.001$ & $0.003 \pm 0.006$ & $7.721 \pm 0.006$ \\
\hline 2002 & $34.920 \pm 0.001$ & $3.273 \pm 0.003$ & $43.1 \pm 0.1$ & $11.06 \pm 0.04$ & $7.727 \pm 0.001$ & $-0.013 \pm 0.006$ & $7.714 \pm 0.006$ \\
\hline 2003 & $34.926 \pm 0.001$ & $3.387 \pm 0.005$ & $45.2 \pm 0.3$ & $11.79 \pm 0.08$ & $7.714 \pm 0.001$ & $0.000 \pm 0.006$ & $7.714 \pm 0.006$ \\
\hline 2004 & $34.909 \pm 0.001$ & $3.226 \pm 0.003$ & $43.8 \pm 0.1$ & $11.32 \pm 0.04$ & $7.722 \pm 0.001$ & $-0.011 \pm 0.006$ & $7.711 \pm 0.006$ \\
\hline 2006 & $34.919 \pm 0.001$ & $3.297 \pm 0.003$ & $42.2 \pm 0.1$ & $11.39 \pm 0.04$ & $7.722 \pm 0.001$ & $-0.008 \pm 0.006$ & $7.714 \pm 0.006$ \\
\hline 2008 & $34.923 \pm 0.001$ & $3.299 \pm 0.003$ & $42.6 \pm 0.1$ & $11.50 \pm 0.04$ & $7.722 \pm 0.001$ & $-0.006 \pm 0.006$ & $7.716 \pm 0.006$ \\
\hline WOA05 & $34.936 \pm 0.001$ & $3.414 \pm 0.012$ & $45.1 \pm 0.1$ & $11.67 \pm 0.05$ & $*$ & * & $*$ \\
\hline \multicolumn{8}{|c|}{ uNADW $\left(\sigma_{2}>37.00 \mathrm{~kg} \mathrm{~m}^{-3} ; \sigma_{4}<45.84 \mathrm{~kg} \mathrm{~m}^{-3}\right)$} \\
\hline 1981 & $34.970 \pm 0.002$ & $2.732 \pm 0.007$ & $52.9 \pm 0.3$ & $10.74 \pm 0.10$ & $7.739 \pm 0.001$ & $0.007 \pm 0.006$ & $7.746 \pm 0.007$ \\
\hline 1991 & $34.972 \pm 0.001$ & $2.762 \pm 0.004$ & $53.0 \pm 0.2$ & $14.66 \pm 0.05$ & $7.739 \pm 0.001$ & $0.006 \pm 0.006$ & $7.745 \pm 0.007$ \\
\hline 1991 & $34.963 \pm 0.001$ & $2.718 \pm 0.005$ & $55.2 \pm 0.2$ & $18.44 \pm 0.07$ & $7.739 \pm 0.001$ & $0.000 \pm 0.006$ & $7.739 \pm 0.007$ \\
\hline 1993 & $34.971 \pm 0.003$ & $2.816 \pm 0.012$ & $49.3 \pm 0.6$ & $12.95 \pm 0.18$ & $7.741 \pm 0.001$ & $0.006 \pm 0.006$ & $7.747 \pm 0.007$ \\
\hline 1997 & $34.949 \pm 0.001$ & $2.689 \pm 0.004$ & $50.0 \pm 0.2$ & $15.53 \pm 0.06$ & $7.733 \pm 0.001$ & $0.003 \pm 0.006$ & $7.736 \pm 0.007$ \\
\hline 1998 & $34.967 \pm 0.004$ & $2.749 \pm 0.017$ & $49.7 \pm 0.9$ & $13.69 \pm 0.26$ & $7.721 \pm 0.002$ & $0.010 \pm 0.006$ & $7.731 \pm 0.007$ \\
\hline 2002 & $34.964 \pm 0.001$ & $2.704 \pm 0.004$ & $52.8 \pm 0.2$ & $15.52 \pm 0.05$ & $7.727 \pm 0.001$ & $0.007 \pm 0.006$ & $7.734 \pm 0.007$ \\
\hline 2003 & $34.975 \pm 0.003$ & $2.833 \pm 0.012$ & $48.3 \pm 0.6$ & $13.51 \pm 0.18$ & $7.715 \pm 0.001$ & $0.007 \pm 0.006$ & $7.722 \pm 0.007$ \\
\hline 2004 & $34.959 \pm 0.001$ & $2.675 \pm 0.004$ & $56.0 \pm 0.2$ & $17.09 \pm 0.06$ & $7.724 \pm 0.001$ & $0.002 \pm 0.006$ & $7.726 \pm 0.007$ \\
\hline 2006 & $34.964 \pm 0.001$ & $2.705 \pm 0.003$ & $53.2 \pm 0.2$ & $17.54 \pm 0.05$ & $7.724 \pm 0.001$ & $0.007 \pm 0.006$ & $7.731 \pm 0.007$ \\
\hline 2008 & $34.964 \pm 0.001$ & $2.701 \pm 0.004$ & $52.3 \pm 0.2$ & $16.37 \pm 0.06$ & $7.722 \pm 0.001$ & $0.010 \pm 0.006$ & $7.731 \pm 0.007$ \\
\hline WOA05 & $34.963 \pm 0.004$ & $2.710 \pm 0.025$ & $56.5 \pm 0.3$ & $18.77 \pm 0.77$ & $*$ & $*$ & $*$ \\
\hline
\end{tabular}


Table 2c. ENA Basin.

\begin{tabular}{|c|c|c|c|c|c|c|c|}
\hline Year & Salinity & $\begin{array}{l}\theta \\
\left({ }^{\circ} \mathrm{C}\right)\end{array}$ & $\begin{array}{l}\text { AOU } \\
\left(\mu \mathrm{mol} \mathrm{kg}{ }^{-1}\right)\end{array}$ & 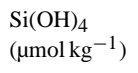 & $\mathrm{pH}_{\mathrm{SWS} 25}$ & $\Delta \mathrm{pH}_{\mathrm{SWS} 25-\mathrm{BA}}$ & $\mathrm{pH}_{\mathrm{SWS25-BA}}$ \\
\hline \multicolumn{8}{|c|}{$\mathrm{NACW}\left(\sigma_{0}<27.20 \mathrm{~kg} \mathrm{~m}^{-3}\right)$} \\
\hline 1981 & $35.618 \pm 0.001$ & $12.472 \pm 0.003$ & $27.5 \pm 0.2$ & $3.74 \pm 0.05$ & $7.883 \pm 0.001$ & $0.004 \pm 0.004$ & $7.887 \pm 0.004$ \\
\hline 1989 & $35.661 \pm 0.001$ & $12.448 \pm 0.004$ & $23.6 \pm 0.2$ & $.86 \pm 0.06$ & $7.871 \pm 0.001$ & $-0.001 \pm 0.004$ & $7.871 \pm 0.004$ \\
\hline 1990 & $.668 \pm 0.001$ & $12.160 \pm 0.003$ & $19.7 \pm 0.1$ & $52 \pm 0.04$ & $7.874 \pm 0.001$ & $0.001 \pm 0.004$ & $7.875 \pm 0.004$ \\
\hline 1991 & $.532 \pm 0.004$ & $11.163 \pm 0.015$ & $22.5 \pm 0.8$ & $.66 \pm 0.23$ & $7.860 \pm 0.002$ & $0.013 \pm 0.004$ & $7.873 \pm 0.004$ \\
\hline 1993 & $544 \pm 0.001$ & $11.500 \pm 0.004$ & $23.0 \pm 0.2$ & $.02 \pm 0.07$ & $7.866 \pm 0.001$ & $0.010 \pm 0.004$ & $7.876 \pm 0.004$ \\
\hline 1997 & $.673 \pm 0.000$ & $2.375 \pm 0.002$ & $.6 \pm 0.1$ & $88 \pm 0.03$ & $7.861 \pm 0.001$ & & $7.871 \pm 0.004$ \\
\hline 1998 & $.659 \pm 0.001$ & $12.293 \pm 0.002$ & $.9 \pm 0.1$ & & & $-0.001 \pm 0.004$ & $7.874 \pm 0.004$ \\
\hline 2002 & & & .1 & & & & \\
\hline 2003 & $.657 \pm 0.000$ & $492 \pm 0.0$ & $1 \pm$ & & 01 & $0.001 \pm 0.0$ & $7.863 \pm 0.004$ \\
\hline 2004 & 0.000 & 0.002 & .1 & & & & $7.864 \pm 0.004$ \\
\hline 2006 & 0.000 & 0.002 & $1 \pm$ & & .001 & 0.004 & $7.857 \pm 0.004$ \\
\hline 2008 & & $22 \pm 0.002$ & .1 & & $7.861 \pm$ & -0. & $7.859 \pm 0.004$ \\
\hline WOA05 & $5.552 \pm 0.004$ & $11.832 \pm 0.029$ & $17.9 \pm 0.3$ & $3.47 \pm 0.02$ & $*$ & * & * \\
\hline \multicolumn{8}{|c|}{ MW $\left(\sigma_{0}>27.20 \mathrm{~kg} \mathrm{~m}^{-3} ; \sigma_{1}<32.35 \mathrm{~kg} \mathrm{~m}^{-3}\right)$} \\
\hline 1981 & & $7.812 \pm 0.003$ & $72.4 \pm 0.1$ & $10.29 \pm 0.04$ & $7.761 \pm 0.001$ & & \\
\hline 1989 & $.745 \pm 0.001$ & $79 \pm 0.003$ & $4 \pm$ & $34 \pm 0$ & $7.781 \pm 0.001$ & $9 \pm 0.002$ & $7.772 \pm 0.002$ \\
\hline 1990 & $.250 \pm 0.001$ & & 6 & & & & \pm 0.002 \\
\hline 1991 & $.107 \pm 0.001$ & $6.650 \pm 0.003$ & $.1 \pm 0.2$ & $10.07 \pm 0.05$ & $7.756 \pm 0.001$ & $0.015 \pm 0.002$ & $7.772 \pm 0.002$ \\
\hline 1993 & $35.242 \pm 0.001$ & $7.125 \pm 0.003$ & $64.9 \pm 0.1$ & $9.08 \pm 0.04$ & $7.754 \pm 0.001$ & $0.019 \pm 0.002$ & $7.774 \pm 0.002$ \\
\hline 1997 & $.509 \pm 0.000$ & $8.413 \pm 0.002$ & $75.4 \pm 0.1$ & $9.77 \pm 0.02$ & $7.759 \pm 0.001$ & $0.010 \pm 0.002$ & $7.770 \pm 0.002$ \\
\hline 1998 & $35.323 \pm 0.001$ & $7.542 \pm 0.003$ & $74.6 \pm 0.2$ & $10.46 \pm 0.05$ & $7.744 \pm 0.001$ & $0.023 \pm 0.002$ & $7.767 \pm 0.002$ \\
\hline 2002 & $35.490 \pm 0.000$ & $8.262 \pm 0.001$ & $74.2 \pm 0.1$ & $9.66 \pm 0.02$ & $7.756 \pm 0.001$ & $0.011 \pm 0.002$ & $7.767 \pm 0.002$ \\
\hline 2003 & $35.365 \pm 0.001$ & $7.771 \pm 0.002$ & $78.5 \pm 0.1$ & $10.21 \pm 0.03$ & $7.743 \pm 0.001$ & $0.023 \pm 0.002$ & $7.766 \pm 0.002$ \\
\hline 2004 & $35.456 \pm 0.000$ & $8.038 \pm 0.001$ & $75.2 \pm 0.1$ & $9.86 \pm 0.02$ & $7.751 \pm 0.001$ & $0.015 \pm 0.002$ & $7.766 \pm 0.002$ \\
\hline 2006 & & & & & & $0.012 \pm 0.002$ & \\
\hline 2008 & $35.445 \pm 0.000$ & $7.998 \pm 0.001$ & $70.6 \pm 0.1$ & $9.69 \pm 0.02$ & $7.751 \pm 0.001$ & $0.010 \pm 0.002$ & $7.761 \pm 0.002$ \\
\hline WOA05 & $35.395 \pm 0.004$ & $8.176 \pm 0.037$ & $60.2 \pm 0.7$ & $9.28 \pm 0.05$ & $*$ & $*$ & $*$ \\
\hline
\end{tabular}

\begin{tabular}{|c|c|c|c|c|c|c|c|}
\hline \multicolumn{8}{|c|}{$\operatorname{LSW}\left(\sigma_{1}>32.35 \mathrm{~kg} \mathrm{~m}^{-3} ; \sigma_{2}<37.00 \mathrm{~kg} \mathrm{~m}^{-3}\right)$} \\
\hline 1981 & $35.057 \pm 0.001$ & $3.975 \pm 0.004$ & $56.0 \pm 0.2$ & $15.15 \pm 0.06$ & $7.746 \pm 0.001$ & $0.003 \pm 0.004$ & $7.749 \pm 0.004$ \\
\hline 1989 & $35.107 \pm 0.001$ & $4.353 \pm 0.006$ & $62.7 \pm 0.3$ & $16.90 \pm 0.09$ & $7.734 \pm 0.001$ & $0.004 \pm 0.004$ & $7.737 \pm 0.004$ \\
\hline 1990 & $35.002 \pm 0.002$ & $3.712 \pm 0.006$ & $54.5 \pm 0.3$ & $14.41 \pm 0.10$ & $7.734 \pm 0.001$ & $0.013 \pm 0.004$ & $7.747 \pm 0.004$ \\
\hline 1991 & $34.920 \pm 0.001$ & $3.286 \pm 0.004$ & $45.4 \pm 0.2$ & $13.21 \pm 0.06$ & $7.743 \pm 0.001$ & $-0.003 \pm 0.004$ & $7.739 \pm 0.004$ \\
\hline 1993 & $34.946 \pm 0.001$ & $3.422 \pm 0.004$ & $47.2 \pm 0.2$ & $12.33 \pm 0.05$ & $7.743 \pm 0.001$ & $-0.002 \pm 0.004$ & $7.740 \pm 0.004$ \\
\hline 1997 & $34.997 \pm 0.001$ & $3.673 \pm 0.002$ & $55.0 \pm 0.1$ & $15.27 \pm 0.03$ & $7.734 \pm 0.001$ & $0.007 \pm 0.004$ & $7.740 \pm 0.004$ \\
\hline 1998 & $34.962 \pm 0.001$ & $3.515 \pm 0.004$ & $50.2 \pm 0.2$ & $13.94 \pm 0.05$ & $7.730 \pm 0.001$ & $0.003 \pm 0.004$ & $7.733 \pm 0.004$ \\
\hline 2002 & $34.990 \pm 0.001$ & $3.636 \pm 0.002$ & $51.9 \pm 0.1$ & $14.01 \pm 0.03$ & $7.732 \pm 0.001$ & $-0.004 \pm 0.004$ & $7.729 \pm 0.004$ \\
\hline 2003 & $34.957 \pm 0.001$ & $3.466 \pm 0.003$ & $52.6 \pm 0.2$ & $13.60 \pm 0.05$ & $7.734 \pm 0.001$ & $-0.004 \pm 0.004$ & $7.730 \pm 0.004$ \\
\hline 2004 & $34.986 \pm 0.000$ & $3.606 \pm 0.002$ & $53.0 \pm 0.1$ & $14.01 \pm 0.02$ & $7.730 \pm 0.001$ & $0.003 \pm 0.004$ & $7.732 \pm 0.004$ \\
\hline 2006 & $34.989 \pm 0.000$ & $3.642 \pm 0.002$ & $50.7 \pm 0.1$ & $13.54 \pm 0.03$ & $7.729 \pm 0.001$ & $-0.001 \pm 0.004$ & $7.728 \pm 0.004$ \\
\hline 2008 & $34.994 \pm 0.000$ & $3.657 \pm 0.002$ & $51.8 \pm 0.1$ & $14.00 \pm 0.03$ & $7.731 \pm 0.001$ & $-0.005 \pm 0.004$ & $7.727 \pm 0.004$ \\
\hline & & & & & & & \\
\hline
\end{tabular}

$\begin{array}{rrrrr}\text { WOA05 } & 34.990 \pm 0.005 & 3.673 \pm 0.031 & 55.4 \pm 0.4 & 14.99 \pm 0.13\end{array}$

\begin{tabular}{|c|c|c|c|c|c|c|c|}
\hline 1981 & $34.947 \pm 0.001$ & $2.610 \pm 0.005$ & $71.7 \pm 0.2$ & $30.40 \pm 0.07$ & $7.738 \pm 0.001$ & $-0.011 \pm 0.003$ & $7.727 \pm 0.003$ \\
\hline 1989 & $34.959 \pm 0.001$ & $2.738 \pm 0.005$ & $74.7 \pm 0.3$ & $31.98 \pm 0.08$ & $7.726 \pm 0.001$ & $-0.009 \pm 0.003$ & $7.718 \pm 0.003$ \\
\hline 1990 & $34.947 \pm 0.002$ & $2.571 \pm 0.009$ & $75.9 \pm 0.5$ & $32.14 \pm 0.14$ & $7.710 \pm 0.001$ & $0.008 \pm 0.003$ & $7.718 \pm 0.003$ \\
\hline 1991 & $34.943 \pm 0.001$ & $2.574 \pm 0.004$ & $68.5 \pm 0.2$ & $28.34 \pm 0.06$ & $7.738 \pm 0.001$ & $-0.020 \pm 0.003$ & $7.718 \pm 0.003$ \\
\hline 1993 & $34.945 \pm 0.001$ & $2.585 \pm 0.006$ & $69.4 \pm 0.3$ & $28.53 \pm 0.08$ & $7.740 \pm 0.001$ & $-0.017 \pm 0.003$ & $7.724 \pm 0.003$ \\
\hline 1997 & $34.944 \pm 0.001$ & $2.597 \pm 0.003$ & $76.3 \pm 0.1$ & $32.62 \pm 0.04$ & $7.733 \pm 0.001$ & $-0.006 \pm 0.003$ & $7.727 \pm 0.003$ \\
\hline 1998 & $34.941 \pm 0.001$ & $2.564 \pm 0.005$ & $72.6 \pm 0.2$ & $31.22 \pm 0.07$ & $7.731 \pm 0.001$ & $-0.012 \pm 0.003$ & $7.719 \pm 0.003$ \\
\hline 2002 & $34.948 \pm 0.000$ & $2.611 \pm 0.002$ & $71.9 \pm 0.1$ & $30.97 \pm 0.03$ & $7.733 \pm 0.001$ & $-0.014 \pm 0.003$ & $7.719 \pm 0.003$ \\
\hline 2003 & $34.938 \pm 0.001$ & $2.515 \pm 0.005$ & $77.3 \pm 0.2$ & $31.52 \pm 0.07$ & $7.720 \pm 0.001$ & $-0.001 \pm 0.003$ & $7.719 \pm 0.003$ \\
\hline 2004 & $34.943 \pm 0.000$ & $2.588 \pm 0.002$ & $73.8 \pm 0.1$ & $31.17 \pm 0.03$ & $7.732 \pm 0.001$ & $-0.012 \pm 0.003$ & $7.719 \pm 0.003$ \\
\hline 2006 & $34.950 \pm 0.000$ & $2.626 \pm 0.002$ & $70.5 \pm 0.1$ & $30.81 \pm 0.03$ & $7.732 \pm 0.001$ & $-0.016 \pm 0.003$ & $7.716 \pm 0.003$ \\
\hline 2008 & $34.945 \pm 0.000$ & $2.597 \pm 0.002$ & $72.0 \pm 0.1$ & $31.78 \pm 0.03$ & $7.734 \pm 0.001$ & $-0.018 \pm 0.003$ & $7.716 \pm 0.003$ \\
\hline WOA05 & $34.944 \pm 0.001$ & $2.589 \pm 0.013$ & $77.9 \pm 0.2$ & $33.02 \pm 0.32$ & & & $*$ \\
\hline \multicolumn{8}{|c|}{ INADW $\left(\sigma_{4}>45.84 \mathrm{~kg} \mathrm{~m}^{-3}\right)$} \\
\hline 1981 & $34.907 \pm 0.001$ & $2.151 \pm 0.006$ & $85.2 \pm 0.3$ & $43.67 \pm 0.09$ & $7.728 \pm 0.001$ & $-0.001 \pm 0.004$ & $7.726 \pm 0.004$ \\
\hline 1989 & $34.905 \pm 0.001$ & $2.130 \pm 0.005$ & $85.2 \pm 0.3$ & $44.98 \pm 0.08$ & $7.719 \pm 0.001$ & $0.000 \pm 0.004$ & $7.719 \pm 0.004$ \\
\hline 1990 & $34.909 \pm 0.002$ & $1.964 \pm 0.009$ & $90.3 \pm 0.4$ & $44.37 \pm 0.13$ & $7.723 \pm 0.001$ & $0.003 \pm 0.004$ & $7.727 \pm 0.004$ \\
\hline 1991 & $34.910 \pm 0.002$ & $2.182 \pm 0.008$ & $87.5 \pm 0.4$ & $44.53 \pm 0.11$ & $7.728 \pm 0.001$ & $-0.002 \pm 0.004$ & $7.726 \pm 0.004$ \\
\hline 1993 & $34.915 \pm 0.001$ & $2.193 \pm 0.005$ & $84.5 \pm 0.3$ & $42.31 \pm 0.08$ & $7.735 \pm 0.001$ & $-0.002 \pm 0.004$ & $7.732 \pm 0.004$ \\
\hline 1997 & $34.904 \pm 0.001$ & $2.131 \pm 0.003$ & $87.4 \pm 0.2$ & $44.33 \pm 0.05$ & $7.732 \pm 0.001$ & $-0.001 \pm 0.004$ & $7.731 \pm 0.004$ \\
\hline 1998 & $34.911 \pm 0.001$ & $2.180 \pm 0.005$ & $87.0 \pm 0.2$ & $43.98 \pm 0.07$ & $7.728 \pm 0.001$ & $-0.002 \pm 0.004$ & $7.726 \pm 0.004$ \\
\hline 2002 & $34.911 \pm 0.001$ & $2.158 \pm 0.002$ & $86.0 \pm 0.1$ & $44.54 \pm 0.03$ & $7.731 \pm 0.001$ & $-0.002 \pm 0.004$ & $7.729 \pm 0.004$ \\
\hline 2003 & $34.912 \pm 0.001$ & $2.192 \pm 0.005$ & $85.6 \pm 0.2$ & $42.05 \pm 0.07$ & $7.724 \pm 0.001$ & $-0.002 \pm 0.004$ & $7.721 \pm 0.004$ \\
\hline 2004 & $34.906 \pm 0.001$ & $2.149 \pm 0.002$ & $87.0 \pm 0.1$ & $44.12 \pm 0.03$ & $7.732 \pm 0.001$ & $-0.002 \pm 0.004$ & $7.730 \pm 0.004$ \\
\hline 2006 & $34.914 \pm 0.001$ & $2.159 \pm 0.002$ & $85.2 \pm 0.1$ & $45.53 \pm 0.03$ & $7.732 \pm 0.001$ & $-0.002 \pm 0.004$ & $7.730 \pm 0.004$ \\
\hline 2008 & $34.909 \pm 0.001$ & $2.159 \pm 0.002$ & $85.4 \pm 0.1$ & $44.94 \pm 0.04$ & $7.734 \pm 0.001$ & $-0.002 \pm 0.004$ & $7.732 \pm 0.004$ \\
\hline WOA05 & $34.908 \pm 0.001$ & $2.101 \pm 0.046$ & $88.7 \pm 0.5$ & $42.96 \pm 0.63$ & $*$ & $*$ & $*$ \\
\hline
\end{tabular}



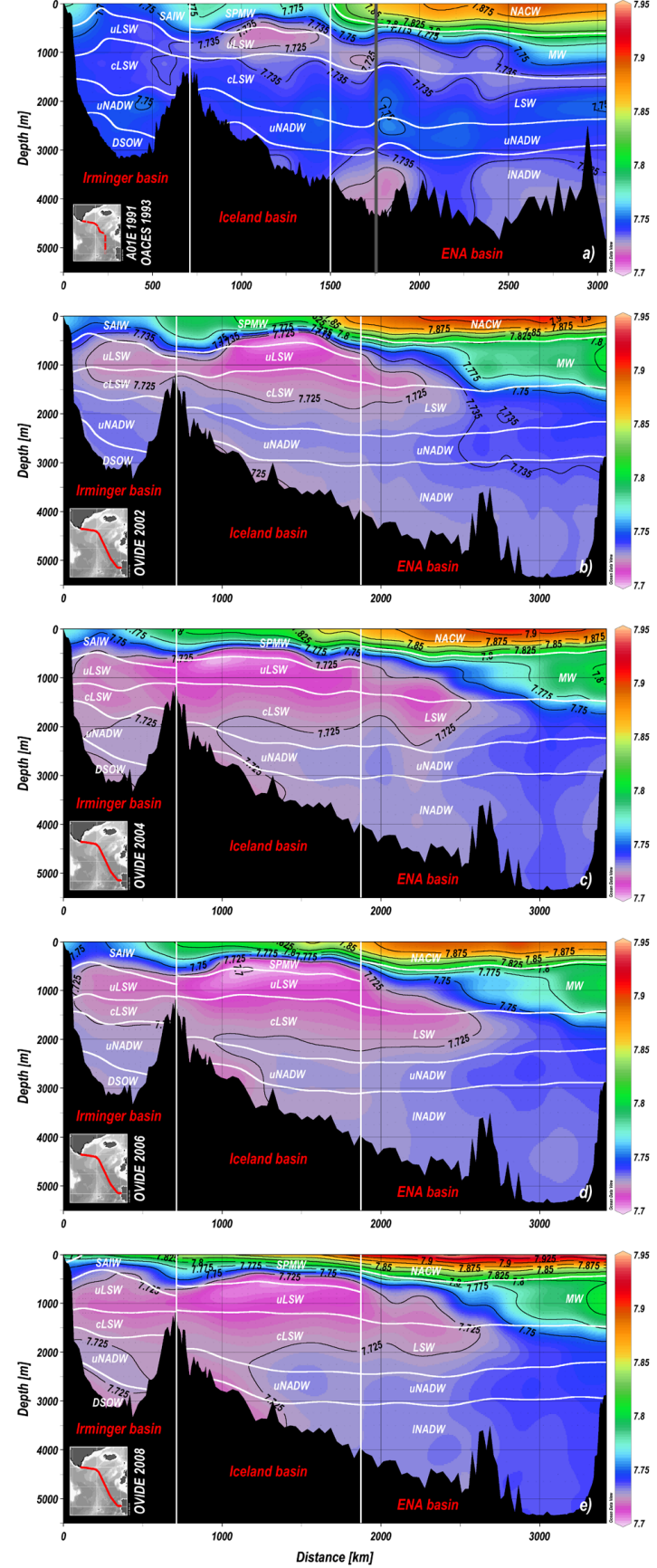

Fig. 2. Evolution of measured $\mathrm{pH}_{\mathrm{SWS} 25}$ distributions in the NASPG from 1991 to 2008. The transect distances $(\mathrm{km})$ from the southernmost tip of Greenland towards the ENA Basin are in the x-axes. The vertical white lines demarcate the transition from basin to basin, as in Fig. 1. The thick horizontal white lines are the isopycnals shown in Fig. 1b and listed on Table 2. Water mass acronyms are the same as in Fig. 1b. The transect represented in (a) is a composite of the A01E and OACES tracks that matches closely the OVIDE section. The two-year difference between the A01E (1991) and OACES (1993) is negligible compared with the nine-year gap between this composite section and the OVIDE 2002 cruise (b), as demonstrated by the continuity of the $\mathrm{pH}$ isopleths at the grey vertical line (merging point between the A01E and OACES tracks). although there exist slight differences between the upper and lower NADW branches (uNADW and INADW). The branch of uNADW that spreads westward into the Iceland Basin mixes with LSW (Yashayaev etl al., 2008) forming a pH gradient that shows decreasing $\mathrm{pH}$ values over time. The influence of LSW in the UNADW is also revealed by the AOU and $\mathrm{Si}(\mathrm{OH})_{4}$ values of the uNADW, which are lower than those in the INADW layer (Table 2c). In the Irminger, Basin the decreasing trends of $\mathrm{pH}_{\mathrm{SWS} 25}$ are clearly visible in the most recently ventilated waters like the uLSW and DSOW (Fig. 2). The latter shows low pH $\mathrm{H}_{\text {SwS25 }}$ in 2004 and 2008 and higher values in 2006 due to the different NAO conditions (Pérez et al., 2010). The most evident sign of acidification is detected between 1000 and $2000 \mathrm{~m}$ depth, where the volume of water with $\mathrm{pH}$ values below 7.725 thickens over time.

To estimate the acidification rates of the water masses, we normalised the discrete in situ $\mathrm{pH}_{S W S 25}$ data to basin-average conditions ( $\left.\mathrm{pH}_{\mathrm{SWS} 25-\mathrm{BA}}\right)$, as described in Sect. 2.2. The average correction $\left(\triangle \mathrm{pH}_{\mathrm{SWS} 25-\mathrm{BA}}\right)$ applied to the studied region is $0.003 \pm 0.009$ (Table 2). On average, the largest corrections correspond to the Irminger Basin $(0.007 \pm 0.009)$, while in the Iceland and ENA Basins they are smaller $(0.003 \pm 0.009$ and $0.002 \pm 0.010$, respectively). In the Irminger Basin no correction was applied to the uNADW and DSOW layers (Table 2a). The highest average corrections in this basin were applied to the uLSW $(0.014 \pm 0.008)$ and cLSW $(0.012 \pm 0.005)$ layers, and the highest individual correction $(0.027 \pm 0.003)$ corresponds to the SAIW in 1997. The smallest average $\mathrm{pH}_{\mathrm{SWS} 25-\mathrm{BA}}$ corrections in the Iceland Basin correspond to the $\mathrm{uLSW}(0.000 \pm 0.003)$ and the largest to the SPMW layer $(0.008 \pm 0.014)$, to which also the highest individual correction was applied $(0.003 \pm 0.005)$, corresponding to the 1991 A01E cruise. In the ENA Basin the smallest average corrections correspond to the LSW and NACW layers $(0.0012 \pm 0.004$ and $0.0045 \pm 0.004$, respectively) and the largest to the MW $(0.014 \pm 0.002)$, where the highest individual corrections were also applied $(0.023 \pm 0.002)$ in 1998 and 2003, both cruises conducted along meridian $20^{\circ} \mathrm{W}$.

In general, we can see a trend of decreasing $\mathrm{pH}$ over time for both $\mathrm{pH}_{\mathrm{SWS} 25}$ and $\mathrm{pH}_{\mathrm{SWS} 25-\mathrm{BA}}$ in all basins and layers (Table 2). These decreasing $\mathrm{pH}_{\mathrm{SWS} 25}$ trends tend to be more pronounced in the Irminger and Iceland Basins and less marked in the ENA Basin (Table 2). The SAIW and uLSW layers in the Irminger Basin show strong decreasing $\mathrm{pH}_{\mathrm{SWS}} 25$ trends in the period 1981 to 1997 (positive NAO index) and less pronounced ones from 2002 to 2008. In the deepest layers (cLSW, uNADW and DSOW) the $\mathrm{pH}_{\mathrm{SWS} 25}$ trends are lower and there is also a minimum value in 1997, when the NAO phase changed from positive to neutral/negative. Similar $\mathrm{pH}_{\mathrm{SWS} 25}$ trends are observed in the Iceland Basin, with a noticeable decrease from 1981 to 1997 during the high NAO, followed by a slower rate of $\mathrm{pH}_{\mathrm{SWS} 25}$ decrease. Differently, the lowering of $\mathrm{pH}_{\mathrm{SwS} 25}$ in the ENA Basin shows a more continuous and steady trend, with a maximum during 1981 and a minimum in 2006, in the NACW and LSW layers. Also 
Table 3. List of $a_{i}$ coefficients obtained for Eq. (2) using the expression in Eq. (3) in each water mass and basin. Between brackets are the properties associated with each $a_{i}$ coefficient and the corresponding units. All $a_{i}$ coefficients have been scaled up by a factor of $10^{3}$, except for the salinity ones $\left(a_{4}\right)$. The n.s. ("not significant") variables explained very little of the pH variability and weakened the overall MLR fit. They were therefore rejected according to a stepwise method of MLR solving. Estimated errors refer to the error of the MLR fits.

\begin{tabular}{|c|c|c|c|c|c|c|c|}
\hline $\begin{array}{l}\text { Water } \\
\text { Mass }\end{array}$ & $R^{2}$ & $\begin{array}{r}\text { Estimated } \\
\text { error }\end{array}$ & 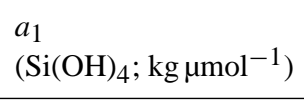 & 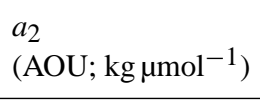 & $\begin{array}{l}a_{3} \\
\left(\theta ;{ }^{\circ} \mathrm{C}^{-1}\right)\end{array}$ & $\begin{array}{l}a_{4} \\
(\mathrm{~S})\end{array}$ & $\begin{array}{l}a_{5} \\
\left(x \mathrm{CO}_{2} ; \mathrm{ppm}^{-1}\right)\end{array}$ \\
\hline \multicolumn{8}{|c|}{ Irminger Basin } \\
\hline SAIW & 0.97 & 0.003 & $-15 \pm 2$ & n.s & n.s & $0.18 \pm 0.03$ & $-1.02 \pm 0.08$ \\
\hline uLSW & 0.99 & 0.002 & $-11 \pm 1$ & n.s & $30 \pm 10$ & $0.67 \pm 0.08$ & $-0.95 \pm 0.04$ \\
\hline cLSW & 0.99 & 0.002 & $-17 \pm 3$ & n.s. & $97 \pm 16$ & $0.44 \pm 0.14$ & $-0.50 \pm 0.04$ \\
\hline uNADW & 0.89 & 0.003 & n.s. & n.s. & n.s. & n.s. & $-0.56 \pm 0.07$ \\
\hline DSOW & 0.78 & 0.005 & n.s. & n.s. & n.s. & n.s. & $-0.57 \pm 0.12$ \\
\hline \multicolumn{8}{|c|}{ Iceland Basin } \\
\hline SPMW & 0.94 & 0.005 & n.s. & $-1.5 \pm 0.3$ & $17 \pm 3$ & n.s. & $-0.61 \pm 0.15$ \\
\hline uLSW & 0.96 & 0.002 & $-7 \pm 2$ & n.s. & n.s. & n.s. & $-0.72 \pm 0.06$ \\
\hline cLSW & 0.81 & 0.006 & $-11 \pm 6$ & n.s. & $-40 \pm 24$ & n.s. & $-0.95 \pm 0.21$ \\
\hline uNADW & 0.75 & 0.006 & n.s. & $2 \pm 1$ & n.s. & $-0.8 \pm 0.4$ & $-0.53 \pm 0.16$ \\
\hline \multicolumn{8}{|c|}{ Eastern North Atlantic Basin } \\
\hline NACW & 0.89 & 0.004 & n.s. & $-1.2 \pm 0.4$ & $11 \pm 3$ & n.s. & $-0.54 \pm 0.09$ \\
\hline MW & 0.96 & 0.002 & n.s. & $-1.0 \pm 0.2$ & $15 \pm 2$ & n.s. & $-0.26 \pm 0.07$ \\
\hline LSW & 0.77 & 0.004 & n.s. & $-0.6 \pm 0.2$ & n.s. & n.s. & $-0.42 \pm 0.08$ \\
\hline uNADW & 0.78 & 0.003 & n.s. & $-2.3 \pm 0.6$ & $27 \pm 11$ & $-3 \pm 1$ & n.s. \\
\hline INADW & 0.28 & 0.004 & n.s. & n.s. & $20 \pm 13$ & n.s. & $0.13 \pm 0.10$ \\
\hline
\end{tabular}

at the ENA Basin, the uNADW and INADW show rather constant $\mathrm{pH}_{\mathrm{SWS} 25}$ values over time, with no clear trends. The $\mathrm{pH}_{\mathrm{SWS} 25}$ signal of the MW layer is noisier due to the important variations in salinity caused by the mixing between MW and other water masses, and as a consequence of changing cruise tracks during the considered time period.

The evolution of the average $\mathrm{pH}_{\mathrm{SWS} 25-\mathrm{BA}}$ between 1981 and 2008 in each layer and basin is plotted in Fig. 3. The error bars on the graph represent the error of the mean and the uncertainty due to the normalization of the data. The general pattern seems to be a decrease of the acidification rates over depth, in all basins. The lowest $\mathrm{pH}_{\mathrm{SWS} 25-\mathrm{BA}}$ vs. time slopes are found in the ENA Basin, while the fastest acidification rates correspond to recently ventilated waters like the SAIW $\left(-0.0019 \pm 0.0001 \mathrm{yr}^{-1}\right)$, the uLSW $(-0.0017 \pm$ $\left.0.00004 \mathrm{yr}^{-1}\right)$ - both of them in the Irminger Basin -, and the SPMW $\left(-0.0012 \pm 0.0002 \mathrm{yr}^{-1}\right)$, in the Iceland Basin. The $\mathrm{pH}_{\mathrm{SWS} 25-\mathrm{BA}}$ of cLSW in the Iceland Basin presents a remarkable average decrease of $-0.0016 \pm 0.0002 \mathrm{yr}^{-1}$, unlike in the Irminger and ENA Basins $(-0.00089 \pm 0.00004$ and $-0.0008 \pm 0.0001 \mathrm{yr}^{-1}$, respectively). The layer of uNADW shows negative $\mathrm{pH}_{\mathrm{SWS} 25-\mathrm{BA}}$ vs. time slopes from the Irminger $\left(-0.0010 \pm 0.0001 \mathrm{yr}^{-1}\right)$ to the Iceland Basin $\left(-0.0008 \pm 0.0002 \mathrm{yr}^{-1}\right)$ due to the influence of ISOW and to the mixing with LSW. Overall, the INADW and UNADW in the ENA Basin are the least acidified water masses over time, with low $\mathrm{pH}_{\mathrm{SWS} 25-\mathrm{BA}}$ vs. time slopes. Their regression fits are, in addition, statistically non-significant (both pvalues $>0.2$ ) and their $\mathrm{pH}_{\mathrm{SWS} 25-\mathrm{BA}}$ vs. time slopes are small, namely: $0.0002 \pm 0.0002 \mathrm{yr}^{-1}\left(R^{2}=0.15 ; \mathrm{p}\right.$-value $\left.=0.57\right)$ and $-0.0003 \pm 0.0001 \mathrm{yr}^{-1}\left(R^{2}=0.28\right.$; $\mathrm{p}$-value $\left.=0.47\right)$ for INADW and uNADW, respectively. The MW in the ENA Basin showed a moderate acidification rate $(-0.0006 \pm$ $0.0001 \mathrm{yr}^{-1}$ ) due to its known capacity for $\mathrm{C}_{\mathrm{ant}}$ drawdown by entrainment from surface layers (Ríos et al., 2001; Álvarez et al., 2005).

\section{Discussion}

The acidification of waters in the upper layer of the NASPG here assessed from in situ $\mathrm{pH}$ measurements spanning the last three decades (1981 to 2008) shows very similar tendencies of $\mathrm{pH}$ decline to those observed in the time series stations ESTOC $\left(29^{\circ} 10^{\prime} \mathrm{N}, 15^{\circ} 30^{\prime} \mathrm{W}\right)$ and BATS $\left(31^{\circ} 43^{\prime} \mathrm{N}, 64^{\circ} 10^{\prime} \mathrm{W}\right)$, in the Subtropical Atlantic. In the Irminger Basin, the observed $\mathrm{pH}_{\mathrm{SWS} 25-\mathrm{BA}}$ decrease rates for SAIW and uLSW are $-0.0019 \pm 0.0002$ and $-0.0017 \pm 0.0001 \mathrm{yr}^{-1}$, respectively, similar to those obtained by Olafsson et al. (2009) for surface waters during the winter $\left(0.0024 \mathrm{yr}^{-1}\right)$. The slight difference with the values reported by Olafsson et al. (2009) likely comes from the fact that the surface isopycnals here considered include thick layers of mode waters with lower interannual variations. The acidification rates here obtained for SAIW and ULSW in the Irminger Basin 


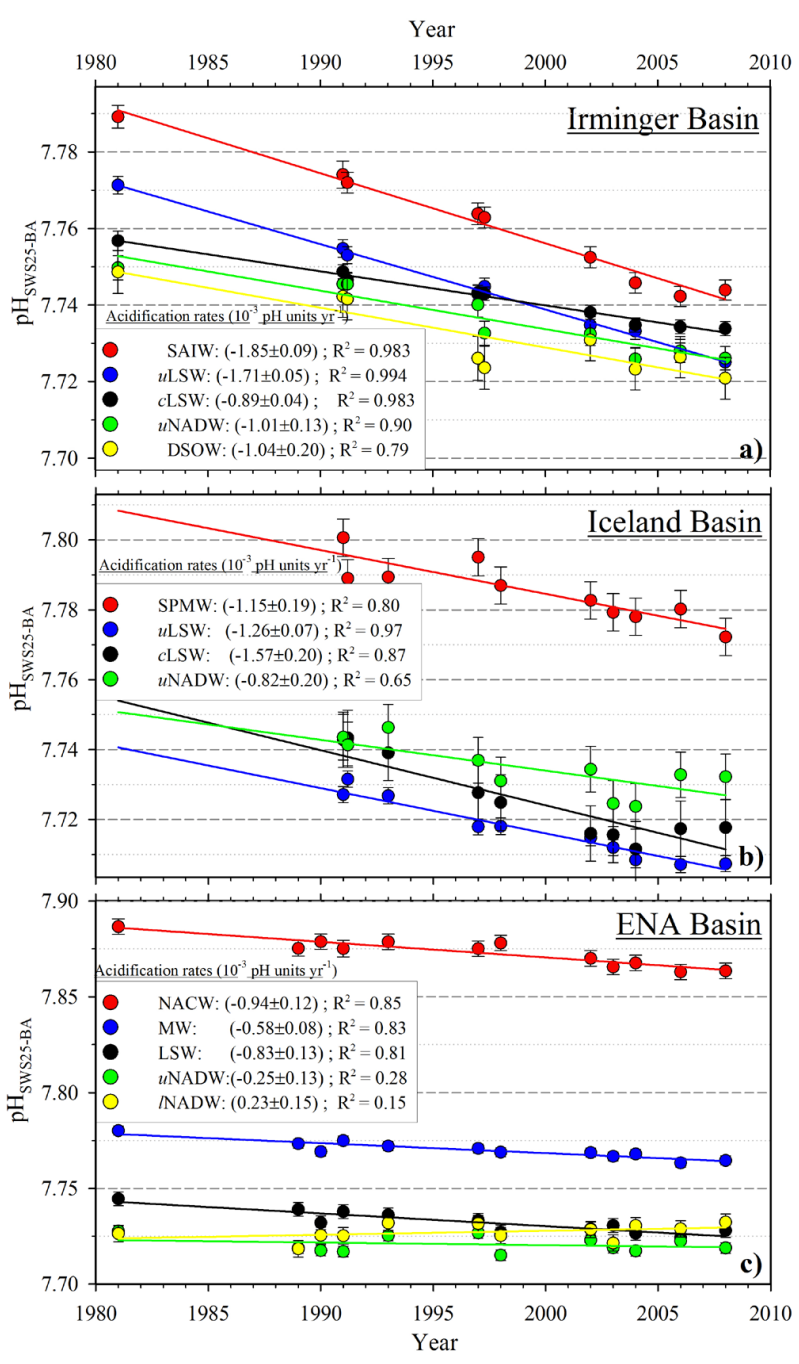

Fig. 3. Trends and rates of acidification between 1981 and 2008 of the studied water masses in the Irminger (a), Iceland (b) and ENA Basins (c). The inset boxes give the acidification rates \pm standard error of the estimate (in $10^{-3} \mathrm{pH}$ units $\mathrm{yr}^{-1}$ ) and correlation coefficients $\left(R^{2}\right)$. Each of the points in the scatter plots represents the average $\mathrm{pH}$ of a particular water mass at the time of the measurement (cruise) $\left(\mathrm{pH}_{\mathrm{SWS} 25-\mathrm{BA}}\right.$; Table 2). Considering the ample time interval of this study (1981-2008), these $\mathrm{pH}$ averages represent well the annual means. The error bars represent the error of the mean plus the uncertainty due to the basin normalization of the data (Sect. 2.2.1).

are also comparable to those reported for the subtropical North Atlantic, at the ESTOC site, in surface waters and in the mixed layer $\left(-0.0017 \mathrm{yr}^{-1}\right)$ during the decade 1995-2004 (Santana-Casiano et al., 2007; González-Dávila et al., 2010), and also at the BATS site, in surface waters $\left(-0.0016 \mathrm{yr}^{-1}\right)$, from 1983 to 2011 (Bates et al., 2012). In the ENA Basin, the decreasing $\mathrm{pH}_{\mathrm{SWS} 25-\mathrm{BA}}$ rate in the NACW $\left(-0.0009 \pm 0.0001 \mathrm{yr}^{-1}\right)$ is similar to those computed at the ESTOC site at 300 and $600 \mathrm{~m}(-0.0010 \pm 0.0004$ and $-0.0008 \pm 0.0003 \mathrm{yr}^{-1}$, respectively) for the decade 1995-2004 (González-Dávila et al., 2010). At $3500 \mathrm{~m}$, the decrease rate of $\mathrm{pH}_{\mathrm{SWS} 25-\mathrm{BA}}$ here obtained for INADW $\left(0.0002 \pm 0.0002 \mathrm{yr}^{-1}\right)$ has a very low $\mathrm{pH}_{\text {SWS25-BA }}$ vs. time correlation coefficient $\left(r^{2}=0.15\right.$; Fig. $\left.3 c\right)$ and is therefore not significant, yet similar to that given by González-Dávila et al. (2010) $\left(-0.0002 \pm 0.0002 \mathrm{yr}^{-1}\right)$ for the same water mass between 1995 and 2004. On the contrary, at the layer where the MW spreads, around $1000 \mathrm{~m}$, González Dávila et al. (2010) reported a $\mathrm{pH}$ decrease rate of $-0.0008 \pm$ $0.0003 \mathrm{yr}^{-1}$, which is slightly higher (considering the associated uncertainties) than our $\mathrm{pH}$ change rate $(-0.0006 \pm$ $0.0001 \mathrm{yr}^{-1}$ ) for the same water mass. This difference could be due to the way MW is defined in our work compared to González-Dávila et al. (2010): they consider MW as a mix of at least three different water types (including MW, Antarctic Intermediate Water and NACW) at the east North Atlantic (González-Dávila et al., 2010).

Ocean uptake and chemical equilibration of $\mathrm{C}_{\text {ant }}$ with seawater results in a gradual reduction of seawater $\mathrm{pH}$ and saturation states $(\Omega)$ for calcium carbonate $\left(\mathrm{CaCO}_{3}\right)$ minerals. However, other contributions to these $\mathrm{pH}$ reductions such as ventilation of the water masses or remineralization of organic matter exist. We have checked whether these obtained $\mathrm{pH}_{\text {SWS25-BA }}$ decrease rates would follow the acidification trends expected mainly from $\mathrm{C}_{\text {ant }}$ uptake contribution, using the $\mathrm{C}_{\mathrm{ant}}$ rates given by Pérez et al. (2010). The necessary $\mathrm{pH}$ values to obtain such rates were calculated using the expression $(\partial \mathrm{pH} / \partial t)_{\mathrm{ANT}}=\left(\partial \mathrm{C}_{\mathrm{ANT}} / \partial t\right)\left(\partial \mathrm{pH} / \partial \mathrm{C}_{\mathrm{T}}\right)_{\left(S, \mathrm{~A}_{\mathrm{T}}\right)}$, where $(\partial \mathrm{pH} / \partial t)_{\mathrm{ANT}}$ is the expected variation over time of anthropogenic $\mathrm{pH}$ (i.e., due to $\left.\mathrm{C}_{\mathrm{ant}}\right)$; $\left(\partial \mathrm{C}_{\mathrm{ANT}} / \partial t\right)$ is the corresponding $\mathrm{C}_{\mathrm{ant}}$ storage rate (from Pérez et al., 2010); and $\left(\partial \mathrm{pH} / \partial \mathrm{C}_{\mathrm{T}}\right)_{\left(S, \mathrm{~A}_{\mathrm{T}}\right)}$ is the variation with respect to $\mathrm{C}_{\mathrm{T}}$ of a $\mathrm{pH}$ calculated from the thermodynamic equations of the marine inorganic carbon system (as described in Sect. 2), using the available $\mathrm{A}_{\mathrm{T}}$ data and salinity measurements.

The $\mathrm{pH}_{\text {SWS25-BA }}$ decrease in the layers of cLSW, uNADW and DSOW (Irminger Basin), and of SPMW and uNADW (Iceland Basin) do follow such expected acidification trend due mainly to $\mathrm{C}_{\text {ant }}$ entry. However, the rest of the considered water masses actually show some deviations from these calculated human-induced acidification patterns. In the layers of uLSW (Irminger and Iceland Basins) and cLSW (Iceland Basin) there is a component $(\sim 50 \%)$ of the observed acidification trends that is not explained by the uptake of $\mathrm{C}_{\mathrm{ant}}$, and is attributed to organic matter remineralization. The SAIW layer in the Irminger Basin presents an intermediate case compared to the previous ones: $\sim 75 \%$ of the $\mathrm{pH}_{\mathrm{SWS} 25-\mathrm{BA}}$ decrease comes from the influence of $\mathrm{C}_{\text {ant }}$. In contrast with what was observed in the Irminger Basin, in the upper layer of the ENA Basin the $\mathrm{C}_{\mathrm{ant}}$-induced acidification is partially compensated by the increase in ventilation (higher $\mathrm{CO}_{2}$ removal via enhanced photosynthetic activity) of the eastern NACW (ENACW) that, overall, produces lower acidification rates than expected. 
From our set of $\mathrm{pH}_{\mathrm{SWS} 25-\mathrm{BA}}$ observations we have made projections of future $\mathrm{pH}$ levels (Fig. 4). The Iceland Basin is particularly suitable for extrapolating the $\mathrm{pH}$ trends from Fig. $3 \mathrm{~b}$ into the future, given the good coverage of measurements available in this region. This characteristic will confer added robustness to the projected acidification trends. The SPMW and cLSW were selected for such projections because out of the considered water bodies, they are some of the most susceptible ones to anthropogenic acidification, and also because they have strong $\mathrm{pH}_{\mathrm{SWS} 25-\mathrm{BA}}$ vs. time fits (Fig. 3b). The projections were calculated under the assumption that the acidification trends in Fig. 3 and the ocean's general circulation behave similarly to what has been observed during the last three decades, for the rest of the 21 st century.

It is known that the strength and the phase of the NAO index affect water mass ventilation and $\mathrm{C}_{\mathrm{ant}}$ uptake rates (Pérez et al., 2010). However, the fact that the NAO phase was close to neutral both during the 1980s and the 2000s should minimise potential biases in the proposed linear projections of $\mathrm{pH}$, which are based on observations from the results here obtained (Fig. 3). Although such linear extrapolation is not constrained, several works have demonstrated that the decline of carbon system parameters, like $\left[\mathrm{CO}_{3}^{2-}\right]$, is almost linear for predictions made between 2000 and 2050 (Zeebe and Wolf-Gladrow, 2001; Hauck et al., 2010). The buffering effect of carbonate minerals and biogenic $\mathrm{CaCO}_{3}$ dissolution can be disregarded since these processes tend to occur in deep waters over timescales that are at least one order of magnitude larger than the one here considered. We can therefore assume that on decadal timescales (our observational time span) $\mathrm{pH}$ will evolve in the future analogously to what we have observed in surface (SPMW) and intermediate (cLSW) waters. This timescale is also within the time frame in which the $x \mathrm{CO}_{2}^{\text {atm }}$ range considered for the $\mathrm{pH}$ projections (Fig. 4) will be reached, under a business-as-usual $\mathrm{CO}_{2}$ emission scenario.

Concerning the assumption of the ocean's general circulation, there is the caveat that the increased stratification of surface layers expected in the future (Friedlingstein and Prentice, 2010) could possibly hamper water mass ventilation processes and potentially bring about a decrease of $\mathrm{pH}$ (acidification), because $\mathrm{C}_{\text {ant }}$ would not be as effectively transported towards the ocean interior via deep convection and water mass formation processes (Pérez et al., 2010). Therefore, if such increased stratification prediction holds true in the future, assuming a steady state for the general circulation can potentially cause overestimates in the $\mathrm{pH}$ values of the linear projections for surface and intermediate waters in Fig. 4. Nevertheless, this acidification slowdown process due to the decrease in $C_{\text {ant }}$ entry could be counterbalanced by the enhanced remineralization rates of organic matter in the upper and intermediate ocean layers that would develop in such a scenario of increased stratification.

According to the obtained $\mathrm{pH}$ projections in Fig. 4, the $\mathrm{pH}$ of surface waters in the Iceland Basin could drop $\sim 0.35$

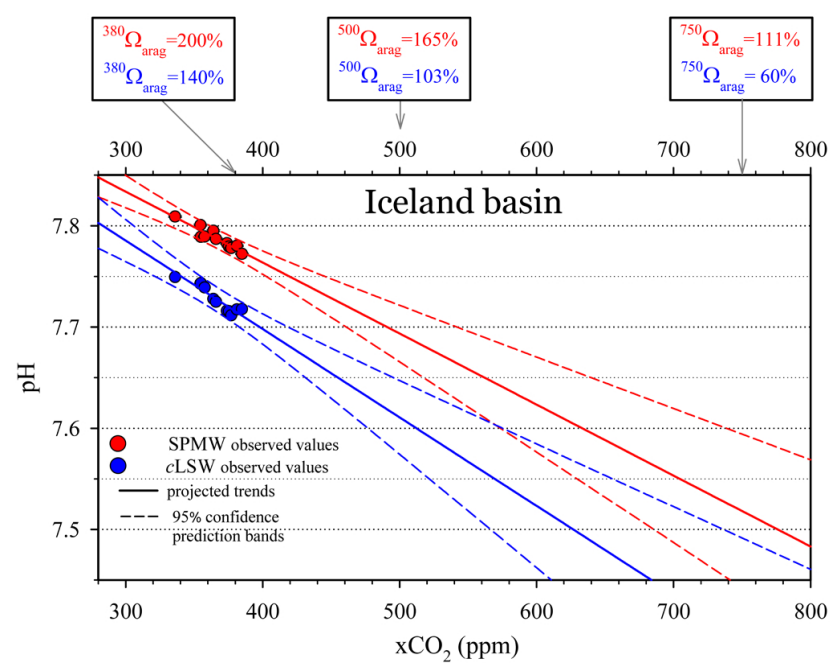

Fig. 4. Extrapolation of the observed linear trends of acidification for the SPMW and cLSW in the Iceland Basin. On the $\mathrm{x}$-axis, the projections range from the pre-industrial $280 \mathrm{ppm}$ to future $800 \mathrm{ppm}$ of atmospheric $x \mathrm{CO}_{2}$ (molar fraction of $\left.\mathrm{CO}_{2}\right)$. The prediction bands give the $95 \%$ confidence intervals for the projected linear trends. The aragonite saturation states ( $\Omega_{\text {arag }}$; as percentages) for present $x \mathrm{CO}_{2}(\sim 380 \mathrm{ppm})$ and for the horizons of 500 and $750 \mathrm{ppm}$ are shown in the top boxes.

units with respect to the pre-industrial era by the time atmospheric $\mathrm{CO}_{2}$ reaches $800 \mathrm{ppm}$, which is consistent with outputs from coupled climate/carbon-cycle models (Caldeira and Wickett, 2005; Orr et al., 2005). In the case of cLSW, the linear projection predicts a $\mathrm{pH}$ decrease of more than 0.45 units with respect to pre-industrial $\mathrm{pH}$ values by the time atmospheric $x \mathrm{CO}_{2}$ reaches $\sim 775 \mathrm{ppm}$ (about twice the present atmospheric concentration of $\mathrm{CO}_{2}$ ). This result is $0.25 \mathrm{pH}$ units lower than the values predicted by the wellknown climate-carbon coupled model in Caldeira and Wickett (2003) for the same $x \mathrm{CO}_{2}^{\text {atm }}$ and ocean region, meaning that according to our results, the cLSW would acidify at a faster rate than expected from numerical simulations. The difference between our observation-based prediction and the latter model (Caldeira and Wickett, 2003) could be due to the fact that our data is largely extrapolated and also that it is still difficult for General Circulation Models (GCMs) to model accurately the Meridional Overturning Circulation (MOC), its NAO-related variability (Danabasoglu et al., 2012) and the deep winter convection of the NASPG. The NAO-related MOC variability has a strong influence on $\mathrm{C}_{\text {ant }}$ storage in the NASPG (Pérez et al., 2010) and it can be therefore expected that this will affect the long-term variability of $\mathrm{pH}$ too, in a way models cannot quite account for yet. In this sense, our results are a good complement and reference for models outputs. On the other hand, different authors have reported that ocean acidification might in fact be proceeding more rapidly than models had predicted (Wootton et al., 2008), as the contemporary $\mathrm{CO}_{2}$ emissions are actually exceeding 
future scenarios based on business-as-usual emission rates (Canadell et al., 2007; Raupach et al., 2007). Such reports are consistent with the lower $\mathrm{pH}$ predictions we obtained compared to Caldeira and Wickett's (2003) results.

The aragonite saturation state is defined as $\Omega_{\text {arag }}=$ $\left[\mathrm{Ca}^{2+}\right]\left[\mathrm{CO}_{3}^{2-}\right] / \mathrm{K}_{\mathrm{sp}}^{\prime}$, where square brackets indicate seawater ion concentrations and $\mathrm{K}_{\mathrm{sp}}^{\prime}$ is the apparent solubility product of aragonite (Mucci, 1983). Because $\left[\mathrm{Ca}^{2+}\right]$ is highly and positively correlated with salinity, $\Omega_{\text {arag }}$ is largely determined by variations in $\left[\mathrm{CO}_{3}^{2-}\right]$. This characteristic makes $\Omega_{\text {arag }}$ an optimum indicator of the environmental availability of dissolved carbonate ions.

From the measured $\mathrm{pH}$ data and our $\mathrm{pH}$ projections (Fig. 4) we calculated the $\Omega_{\text {arag }}$ of the SPMW and cLSW in the Iceland Basin for atmospheric $x \mathrm{CO}_{2}$ values of 380 ( $\sim$ present day), 500 and 750 ppm (see insets in Fig. 4). The results suggest that cLSW would actually reach aragonite undersaturation $\left(\Omega_{\text {arag }}<1\right)$ by the time atmospheric $\mathrm{CO}_{2}$ reaches $\sim 550 \mathrm{ppm}$ and not $900 \mathrm{ppm}$, as suggested by the numerical model predictions in Orr et al. (2005). The highNAO enhanced ventilation that occurred towards the mid-late 1980s fostered the fast formation of a massive vintage of cLSW (Kieke et al., 2007; Yashayaev et al., 2008). The rapid subduction of this newly formed cLSW injected $\mathrm{C}_{\text {ant }}$ from the surface into intermediate waters, transporting $C_{a n t}$ much faster than via downward diffusion alone and thus causing a faster acidification rate in the cLSW (where organic matter remineralization also contributes significantly to the $\mathrm{pH}$ lowering) than in the SPMW, where $\mathrm{C}_{\text {ant }}$ influence is the main contributor to acidification. Depending on the future $\mathrm{CO}_{2}$ emission rates the $550 \mathrm{ppm}$ threshold at which, according to our projections, cLSW would face aragonite undersaturation, could be passed by 2050 or before (Nakicenovic et al., 2000; Caldeira and Wickett, 2005; Feely et al., 2009). Guinotte et al. (2006) have in fact pointed out that some deep-sea coldwater corals may experience undersaturated waters as early as 2020 under an IPCC "business-as-usual" $\mathrm{CO}_{2}$ emission pathway, which is in good agreement with our observationbased results for the Iceland and Irminger Basins.

The data analysis also showed that the aragonite saturation depth (lysocline $=$ isopleth where $\Omega_{\text {arag }}=1$ ) has shoaled at a rate of 7 and $4 \mathrm{~m} \mathrm{yr}^{-1}$ between 1981 and 2008 in the Irminger and Iceland Basins, respectively. The latter is in agreement with previous local studies (Olafsson et al., 2009). The fast rate of lysocline shoaling in the Irminger Basin is promoted by the intense NAO-enhanced deep convection that injects ventilated, $\mathrm{CO}_{2}$-rich waters into deeper ocean layers (Messias et al., 2008), as mentioned previously. For comparison's sake, the shoaling rates of the lysocline were estimated to be $\sim 0.2 \mathrm{~m} \mathrm{yr}^{-1}$ during the Paleocene-Eocene Thermal Maximum (55 million yr ago), when a massive natural release of $\mathrm{CO}_{2}$ into the atmosphere caused global temperatures to raise more than $5^{\circ} \mathrm{C}$ in less than $10000 \mathrm{yr}$ (Pelejero et al., 2010).

\section{Conclusions}

The progressive acidification of North Atlantic waters has been assessed from direct $\mathrm{pH}$ observations spanning the last three decades. The increasing atmospheric $\mathrm{CO}_{2}$ concentrations have largely affected the $\mathrm{pH}$ of surface and intermediate waters in the three studied North Atlantic regions, at varying extents. Most importantly, the LSW has shown very high acidification rates that are amongst the highest found in the NASPG. In the Irminger Basin, the acidification rate of cLSW responds to that expected from the influence of $\mathrm{C}_{\mathrm{ant}}$, while in the Iceland Basin only about $50 \%$ of the observed $\mathrm{pH}$ change in the cLSW is anthropic. The SAIW has the fastest acidification rate observed $\left(-0.0019 \pm 0.0002 \mathrm{yr}^{-1}\right)$, and $75 \%$ of this $\mathrm{pH}$ decrease is anthropogenic. In contrast, the $\mathrm{C}_{\text {ant }}$ contribution to the acidification rates in the ENACW is partially compensated by the ventilation of this water mass, thus explaining the moderate acidification rates observed in the upper layers of the ENA Basin (compared to the Iceland and Irminger Basins). Predictions from an observation-based extrapolation of the current acidification trends and rates are in agreement with model results (Caldeira and Wickett, 2005; Orr et al., 2005) in surface layers. However, our results indicate that the intermediate waters of the North Atlantic (LSW in particular) are getting acidified more rapidly than models predicted.

Acknowledgements. The authors wish to thank C. Pelejero for his comments. The research leading to these results was supported by the EU FP7 project CARBOCHANGE "Changes in carbon uptake and emissions by oceans in a changing climate", which received funding from the European Commission's Seventh Framework Programme under grant agreement no. 264879; by the Spanish Ministry of Science and Innovation and co-funded by Fondo Eurpeo de Desarrollo Regional 2007-2012 (FEDER) through the CATARINA Project (CTM2010-17141). The OVIDE research project was co-funded by the IFREMER, CNRS/INSU and LEFE. H.M. was supported by CNRS. M.V.R. was funded by CSIC I3P Predoctoral Grant program (I3P-BPD2005).

Edited by: L. Bopp

\section{References}

Álvarez, M., Pérez, F. F., Shoosmith, D. R., and Bryden, H. L.: The unaccounted role of Mediterranean Water in the drawdown of anthropogenic carbon, J. Geophys. Res., 110, 1-18, doi:10.1029/2004JC002633, 2005.

Azetsu-Scott, K., Jones, E. P., Yashayaev, I., and Gershey, R. M.: Time series study of CFC concentrations in the Labrador Sea during deep and shallow convection regimes (1991-2000), J. Geophys. Res., 108, 3354, doi:10.1029/2002JC001317, 2003.

Bates, N. R., Best, M. H. P., Neely, K., Garley, R., Dickson, A. G., and Johnson, R. J.: Detecting anthropogenic carbon dioxide uptake and ocean acidification in the North Atlantic Ocean, Biogeosciences, 9, 2509-2522, doi:10.5194/bg-9-2509-2012, 2012. 
Bradshaw, A., Brewer, P., Shafer, D., and Williams, R.: Measurements of total carbon dioxide and alkalinity by potentiometric titration in the GEOSECS program, Earth Planet. Sc. Lett., 55, 99-115, 1981.

Byrne, R. H., Mecking, S., Feely, R. A., and Liu, X.: Direct observations of basin-wide acidification of the North Pacific Ocean, Geophys. Res. Lett., 37, L02601, doi:10.1029/2009GL040999, 2010.

Caldeira, K. and Wickett, M. E.: Anthropogenic carbon and ocean pH, Nature, 425, p. 365, 2003.

Caldeira, K. and Wickett, M. E.: Ocean model predictions of chemistry changes from carbon dioxide emissions to the atmosphere and ocean, J. Geophys. Res., 110, C09S04, doi:10.1029/2004JC002671, 2005.

Canadell, J., LeQuéré, C., Raupach, M. R., Fields, C., Buitenhuis, E. T., Ciais, P., Conway, T. J., Gillett, N. P., Houghton, R. A., and Marland, G.: Contributions to accelerating atmospheric $\mathrm{CO}_{2}$ growth from economic activity, carbon intensity, and efficiency of natural sinks, P. Natl. Acad. Sci., 104, 18866-18870, doi:10.1073/pnas.0702737104, 2007.

Clayton, T. D. and Byrne, R. H.: Calibration of m-cresol purple on the total hydrogen ion concentration scale and its application to $\mathrm{CO}_{2}$-system characteristics in seawater, Deep Sea Res.-Pt. I, 40, 2115-2129, 1993.

Danabasoglu, G., Yeager, S. G., Kwon, Y.-O., Tribbia, J. J., Phillips, A. S., and Hurrell, J. W.: Variability of the atlantic meridional overturning circulation in CCSM4, J. Climate, 25, 5153-5172, doi:10.1175/JCLI-D-11-00463.1, 2012.

Dickson, A. G.: The measurement of sea water pH, Mar. Chem., 44, 131-142, 1993.

Dickson, A. G. and Millero, F. J.: A comparison of the equilibrium constants for the dissociation of carbonic acid in seawater media, Deep Sea Res.-Pt. I, 34, 1733-1743, 1987.

Dickson, A. G., Sabine, C. L., and Christian, J. R. (Eds.): Guide to best practices for ocean $\mathrm{CO}_{2}$ measurements, PICES Special Publication, 3, 191 pp, 2007.

Doney, S. C., Fabry, V. J., Feely, R. A., and Kleypas, J. A.: Ocean Acidification: The Other $\mathrm{CO}_{2}$ Problem, Annu. Rev. Mar., 1, 169192, 2009.

Feely, R. A., Sabine, C. L., Hernandez-Ayon, J. M., Ianson, D., and Hales, B.: Evidence for upwelling of corrosive "acidified" water onto the continental shelf, Science, 320, 1490-1492, 2008.

Feely, R. A., Doney, S. C., and Cooley, S. R.: Ocean acidification: present conditions and future changes in a high- $\mathrm{CO}_{2}$ world, Oceanography, 22, 160-171, 2009.

Friedlingstein, P. and Prentice, I. C.: Carbon-climate feedbacks: a review of model and observation based estimates, Curr. Opin. Environ. Sust., 2, 251-257, doi:10.1016/j.cosust.2010.06.002, 2010.

González-Dávila, M., Santana-Casiano, J. M., Rueda, M. J., and Llinás, O.: The water column distribution of carbonate system variables at the ESTOC site from 1995 to 2004, Biogeosciences, 7, 3067-3081, doi:10.5194/bg-7-3067-2010, 2010.

Guinotte, J. M., Orr, J., Cairns, S., Freiwald, A., Morgan, L., and George, R.: Will human-induced changes in seawater chemistry alter the distribution of deep-sea scleractinian corals?, Front. Ecol. Environ., 4, 141-146, 2006.

Hauck, J., Hoppema, M., Bellerby, R. G. J., Völker, C., and WolfGladrow, D.: Data-based estimation of anthropogenic carbon and acidification in the Weddell Sea on a decadal timescale, J. Geophys. Res., 115, C03004, doi:10.1029/2009JC005479, 2010.

Ilyina, T., Zeebe, R., and Brewer, P.: Changes in underwater sound propagation caused by ocean acidification, IOP Conf. Series: Earth and Environmental Science, 6, 462007, doi:10.1088/17551307/6/6/462007, 2009.

Johnson, K. M., Wills, K. D., Butler, D. B., Johnson, W. K., and Wong, C. S.: Coulometric total carbon dioxide analysis for marine studies: Maximizing the performance of an automated continuous gas extraction system and coulometric detector, Mar. Chem., 44, 167-189, 1993.

Khatiwala, S., Primeau, F., and Hall, T.: Reconstruction of the history of anthropogenic $\mathrm{CO}_{2}$ concentrations in the ocean, Nature, 462, 346-349, doi:10.1038/nature08526, 2009.

Kieke, D., Rhein, M., Stramma, L., Smethie, W. M., Bullister, J. L., and LeBel, D. A.: Changes in the pool of Labrador Sea Water in the subpolar North Atlantic, Geophysical Res. Lett., 34, L06605, doi:10.1029/2006GL028959, 2007.

Lherminier, P., Mercier, H., Huck, T., Gourcuff, C., Perez, F. F., Morin, P., Sarafanov, A., and Falina, A.: The Atlantic Meridional Overturning Circulation and the Subpolar Gyre observed at the A25-OVIDE section in June 2002 and 2004, Deep Sea Res.-Pt. I, 57, 1374-1391, doi:10.1016/j.dsr.2010.07.009, 2010.

McCartney, M. S. and Talley, L. D.: The Subpolar Mode Water of the North Atlantic Ocean, J. Phys. Oceanogr., 12, 1169-1188, 1982.

Messias, M. J., Watson, A. J., Johannessen, T., Oliver, K. I. C., Olsson, K. A., Fogelqvist, E., Olafsson, J., Bacon, S., Balle, J., Bergman, N., Budéus, G., Danielsen, M., Gascard, J. C., Jeansson, E., Olafsdottir, S. R., Simonsen, K., Tanhua, T., Scoy, K. V., and Ledwell, J. R.: The Greenland Sea tracer experiment 19962002: Horizontal mixing and transport of Greenland Sea Intermediate Water, Prog. Oceanogr., 78, 85-105, 2008.

Millero, F. J.: The marine inorganic carbon cycle, Chem. Rev., 107, 308-341, 2007.

Millero, F. J., Zhang, J. Z., Lee, K., and Campbell, D. M.: Titration alkalinity of seawater, Mar. Chem., 44, 153-156, 1993.

Mintrop, L., Perez, F. F., Gonzalez-Davila, M., Santana-Casiano, M. J., and Kortzinger, A.: Alkalinity determination by potentiometry: Intercalibration using three different methods, Cienc. Mar., 26, 23-37, 2002.

Mucci, A.: The solubility of calcite and aragonite in sea water at various salinities, temperatures and one atmosphere total pressure, Am. J. Sci., 238, 780-799, 1983.

Nakicenovic , N., Alcamo, J., Davis, G., de Vries, B., Fenhann, J., Gaffin, S., Gregory, K., Grubler, A., Jung, T. Y., Kram, T., La Rovere, E. L., Michaelis, L., Mori, S., Morita, T., Pepper, W., Pitcher, H., Price, L., Raihi, K., Roehrl, A., Rogner, H.-H., Sankovski, A., Schlesinger, M., Shukla, P., Smith, S., Swart, R., van Rooijen, S., Victor, N., and Dadi, Z.: IPCC Special Report on Emissions Scenarios, Cambridge University Press, Cambridge, United Kingdom and New York, NY, USA, 599 pp., 2000.

Olafsson, J., Olafsdottir, S. R., Benoit-Cattin, A., Danielsen, M., Arnarson, T. S., and Takahashi, T.: Rate of Iceland Sea acidification from time series measurements, Biogeosciences, 6, 26612668, doi:10.5194/bg-6-2661-2009, 2009.

Orr, J. C., Fabry, V. J., Aumont, O., Bopp, L., Doney, S. C., Feely, R. A., Gnanadesikan, A., Gruber, N., Ishida, A., Joos, F., Key, R. M., Lindsay, K., Maier-Reimer, E., Matear, R., Mon- 
fray, P., Mouchet, A., Najjar, R. G., Plattner, G.-K., Rodgers, K. B., Sabine, C. L., Sarmiento, J. L., Schlitzer, R., Slater, R. D., Totterdell, I. J., Weirig, M. F., Yamanaka, Y., and Yool, A.: Anthropogenic ocean acidification over the twenty-first century and its impact on calcifying organisms, Nature, 437, 681-686, doi:10.1038/nature04095, 2005.

Pelejero, C., Calvo, E., Hoegh-Guldberg, O.: Paleo-perspectives on ocean acidification, Trends Ecol. Evol., 25, 332-344, 2010.

Pérez, F. F. and Fraga, F.: A precise and rapid analytical procedure for alkalinity determination, Mar. Chem., 21, 169-182, 1987.

Pérez, F. F., Vázquez-Rodríguez, M., Louarn, E., Padín, X. A., Mercier, H., and Ríos, A. F.: Temporal variability of the anthropogenic $\mathrm{CO}_{2}$ storage in the Irminger Sea, Biogeosciences, 5, 1669-1679, doi:10.5194/bg-5-1669-2008, 2008.

Pérez, F. F., Vázquez-Rodríguez, M., Mercier, H., Velo, A., Lherminier, P., and Ríos, A. F.: Trends of anthropogenic $\mathrm{CO}_{2}$ storage in North Atlantic water masses, Biogeosciences, 7, 1789-1807, doi:10.5194/bg-7-1789-2010, 2010.

Pierrot, D., Brown, P., Van Heuven, S., Tanhua, T., Schuster, U., Wanninkhof, R., and Key, R. M.: CARINA $\mathrm{TCO}_{2}$ data in the Atlantic Ocean, Earth Syst. Sci. Data, 2, 177-187, doi:10.5194/essd-2-177-2010, 2010.

Raupach, M. R., Marland, G., Ciais, P., LeQuéré, C., Canadell, J. G., Klepper, G., and Field, C. B.: Global and regional drivers of accelerating $\mathrm{CO}_{2}$ emissions, P. Natl. Acad. Sci. USA, 104, 10288-10293, 2007.

Raven, J.: Ocean acidification due to increasing atmospheric carbon dioxide, Document No. 12/05, The Royal Society, London, 2005.

Read, J. F.: CONVEX-91: water masses and circulation of the Northeast Atlantic subpolar gyre, Prog. Oceanogr., 48, 461-510, 2001.

Ríos, A. F., Pérez, F. F., and Fraga, F.: Water masses in the upper and middle North Atlantic Ocean east of the Azores, Deep-Sea Res.-Pt. II, 39, 645-658, 1992.

Ríos, A. F., Pérez, F. F., and Fraga, F.: Long term (1977-1997) measurements of carbon dioxide in the Eastern North Atlantic: evaluation of anthropogenic input, Deep-Sea Res.-Pt. II, 48, 22272239, 2001.

Ríos, A. F., Velo, A., Pardo, P. C., Hoppema, M., and Pérez, F. F.: An update of anthropogenic $\mathrm{CO}_{2}$ storage rates in the western South Atlantic basin and the role of Antarctic Bottom Water, J. Marine Syst., 94, 197-203, doi:10.1016/j.jmarsys.2011.11.023, 2012.
Santana-Casiano, J. M., González-Dávila, M., Rueda, M.-J., Llinás, O., and González-Dávila, E.-F.: The interanual variability of oceanic $\mathrm{CO}_{2}$ parameters in the northeast Atlantic subtropical gyre at the ESTOC site, Global Biogeochem. Cy., 21, GB1015, doi:10.1029/2006GB002788, 2007.

Thierry, V., de Boisséson, E., and Mercier, H.: Interannual variability of the Subpolar Mode Water properties over the Reykjanes Ridge during 1990-2006, J. Geophys. Res., 113, C04016, doi:10.1029/2007JC004443, 2008.

Tittensor, D. P., Baco, A. R., Hall-Spencer, J. M., Orr, J. C., and Rogers, A. D.: Seamounts as refugia from ocean acidification for cold-water stony corals, Mar. Ecol., 31, 212-225, doi:10.1111/j.1439-0485.2010.00393.x, 2010.

Velo, A., Perez, F. F., Brown, P., Tanhua, T., Schuster, U., and Key, R. M.: CARINA alkalinity data in the Atlantic Ocean, Earth Syst. Sci. Data, 1, 45-61, doi:10.5194/essd-1-45-2009, 2009.

Velo, A., Pérez, F. F., Lin, X., Key, R. M., Tanhua, T., de la Paz, M., Olsen, A., van Heuven, S., Jutterström, S., and Ríos, A. F.: CARINA data synthesis project: $\mathrm{pH}$ data scale unification and cruise adjustments, Earth Syst. Sci. Data, 2, 133-155, doi:10.5194/essd-2-133-2010, 2010.

Wootton, J. T., Pfister, C. A., and Forester, J. D.: Dynamic patterns and ecological impacts of declining ocean $\mathrm{pH}$ in a highresolution multi-year dataset, P. Natl. Acad. Sci., 105, 1884818853, 2008.

Yashayaev, I., Penny Holliday, N., Bersch, M., and van Aken, H. M.: The history of the Labrador Sea Water: Production, Spreading, Transformation and Loss, in: Arctic-Subarctic Ocean Fluxes: defining the role of the Northern Seas in climate, edited by: Robert, R., Dickson, J., and Meincke, P., Rhines, Springer, P.O. Box 17, 3300 AA Dordrecht, The Netherlands, 569-612, 2008.

Zeebe, R. E. and Wolf-Gladrow, D.: $\mathrm{CO}_{2}$ in Seawater: Equilibrium, Kinetics, Isotopes, Amsterdam: Elsevier Science, B.V. 346 pp., 2001. 